OPEN ACCESS

Edited by:

Reniie Chai,

Southeast University, China

Reviewed by:

Hary Razafindralambo,

ProBioLab, Belgium

Qinrui Li,

Peking University People's Hospital,

China

*Correspondence: Yun Cai

cyayq81@outlook.com

Xiaotang Fan

fanxiaotang2005@163.com

Specialty section:

This article was submitted to Molecular and Cellular Pathology,

a section of the journal

Frontiers in Cell and Developmental

Biology

Received: 10 October 2021

Accepted: 20 January 2022

Published: 03 March 2022

Citation:

Liu J, Gao Z, Liu C, Liu T, Gao J, CaiY and Fan X (2022) Alteration of Gut Microbiota: New Strategy for Treating

Autism Spectrum Disorder.

Front. Cell Dev. Biol. 10:792490.

doi: 10.3389/fcell.2022.792490

\section{Alteration of Gut Microbiota: New Strategy for Treating Autism Spectrum Disorder}

\author{
Jiayin Liu ${ }^{1,2}$, Zhanyuan Gao ${ }^{1,2}$, Chuanqi Liu ${ }^{1,2}$, Tianyao Liu ${ }^{1}$, Junwei Gao ${ }^{1}$, Yun Cai ${ }^{1 *}$ and \\ Xiaotang Fan ${ }^{1 *}$
}

${ }^{1}$ Department of Military Cognitive Psychology, School of Psychology, Third Military Medical University (Army Medical University), Chongqing, China, ${ }^{2}$ Battalion 5th of Cadet Brigade, Third Military Medical University (Army Medical University), Army Medical University, Chongqing, China

Autism spectrum disorder (ASD) is defined as a complex heterogeneous disorder and characterized by stereotyped behavior and deficits in communication and social interactions. The emerging microbial knowledge has pointed to a potential link between gut microbiota dysbiosis and ASD. Evidence from animal and human studies showed that shifts in composition and activity of the gut microbiota may causally contribute to the etiopathogenesis of core symptoms in the ASD individuals with gastrointestinal tract disturbances and act on microbiotagut-brain. In this review, we summarized the characterized gut bacterial composition of ASD and the involvement of gut microbiota and their metabolites in the onset and progression of ASD; the possible underlying mechanisms are also highlighted. Given this correlation, we also provide an overview of the microbial-based therapeutic interventions such as probiotics, antibiotics, fecal microbiota transplantation therapy, and dietary interventions and address their potential benefits on behavioral symptoms of ASD. The precise contribution of altering gut microbiome to treating core symptoms in the ASD needs to be further clarified. It seemed to open up promising avenues to develop microbial-based therapies in ASD.

Keywords: gut microbiota, autism, therapeutic interventions, microbiota-gut-brain, core symptoms

\section{INTRODUCTION}

Autism spectrum disorder (ASD) is defined as a complex heterogeneous disorder with pervasive neurodevelopmental disability (Arakawa, 2020; Valentino et al., 2021). The clinical characteristics of ASD include impairments in social interaction and communication, as well as restricted interests and repetitive behaviors (Supekar et al., 2021). ASD generally appears early in childhood and is diagnosed before the age of three in most cases. The incidence of ASD has dramatically increased in recent years, with an estimated prevalence of $1 \%-2 \%$ according to numerous studies conducted in Asia, Europe, and North America (Elsabbagh et al., 2012; Valentino et al., 2021). Gender ratios have been reported to have a higher prevalence in males than in females, ranging from 2:1 to 5:1 (Brunissen et al., 2021). It is obvious that ASD intervention has become a very urgent need for public health to solve the cost and complexity of medical care. Social skill interventions based on behavioral therapy and pharmacological interventions aimed at comorbidities have been adopted in patients with Asperger's syndrome and high-functioning autism (Corbett et al., 2019; Romagnoli et al., 2019; Frolli et al., 2020; Oshima et al., 2020). However, due to the complexity of the pathogenesis mechanism underlying the disease and limited animal models, there is a lack of Food and Drug Administration (FDA)-approved drug to treat the core symptoms of ASD effectively (Donegan and Lodge, 2020). 
Currently, many disparate mechanisms, such as decline in neurogenesis, an imbalance in excitatory/inhibitory neurotransmission, and dysregulated immune response, have been hypothesized to be involved in the onset and development of ASD-related behavior (Selimbeyoglu et al., 2017; Cai et al., 2018; Cristiano et al., 2018; Cai et al., 2019; Zhang et al., 2019; Dong et al., 2020; Poornimai Abirami et al., 2020; Sacai et al., 2020; Adhya et al., 2021; Zhong et al., 2020; Baranova et al., 2021; Cast et al., 2021). Notably, the emerging microbial knowledge has pointed that the complex intestinal microbial community and their metabolic consequences may contribute to ASD etiopathogenesis of core symptoms, such as in central nervous system (CNS)-driven behaviors, in a very powerful way (Oh and Cheon, 2020). The communication between the brain and the gut is commonly known as the microbiota-gut-brain axis, which is suggested to be involved in maintaining physiological homeostasis (Oh and Cheon, 2020). The bi-directional communication between the gut and brain occurs via neuroimmune and neuroendocrine signaling, the vagus nerve, and gut microbial metabolites (Ersöz Alan and Gülerman, 2019). It is increasingly understood that gut microbiota contributes to the regulation of social behavior via the microbiome-gut-brain axis (Dinan and Cryan, 2017). More recently, several lines of studies suggest that microbial-based therapeutic interventions such as fecal microbiota transplantation (FMT), antibiotics, and probiotics have emerged as novel therapeutic strategies to improve core and associated ASD symptoms (Johnson et al., 2020; MartínezGonzález and Andreo- Martínez, 2020; Davies et al., 2021).

This review article will describe the involvement of gut microbiota and their metabolites in the ASD pathogenesis and progression. Existing literature relevant to therapeutic interventions based on alteration of gut microbiota in ASD appears to fall within the following four domains: prebiotic/ probiotic/synbiotic, FMT, dietary interventions, and antibiotics. The literature on each domain will be reviewed and microbial-based therapeutic interventions in ASD patients will be highlighted in a comprehensive way.

\section{MICROBIOTA-GUT-BRAIN AXIS IN ASD}

The human gut is an integral part of human physiology and metabolism and harbors trillions of microbes including bacteria, eukaryotic microorganisms, viruses, and archaea, commonly referred to as the gut microbiota (Moszak et al., 2020; Ogunrinola et al., 2020). The distinctive microbial signature increases exponentially from the proximal to the distal portion of the gastrointestinal tract (GIT) and varies according to many factors such as age, genetics, and nutrition (Dhar and Mohanty, 2020; Giles and Couper, 2020; Sakkas et al., 2020; Seitz et al., 2020). Moreover, short-chain fatty acids (SCFAs), the microbial metabolites produced by gut bacteria, have been indicated to exert profound effects on the gut, brain, and behavior and contribute to ASD pathology (Tran and Mohajeri, 2021). Notably, SCFAs mainly contain butyrate, propionate, and acetate; possess neuroactive properties; and can operate on immunomodulatory system (Parada Venegas et al., 2019). Gut microbiota, including the four major phyla (Bacteroidetes, Firmicutes, Proteobacteria, and Actinobacteria) and two minor phyla (Verrucomicrobia and Fusobacteria), is increasingly recognized as a metabolic organ, contributing to the maintenance of individual homeostasis through nutrition, immune regulation, and systemic inflammation pathways (Guo et al., 2018; Adak and Khan, 2019; Khan et al., 2020).

An increasing amount of evidence has shown that GIT symptoms, ranging from constipation to diarrhea, are more frequently reported in autistic children than in typically developing (TD) children (Lefter et al., 2019; Sanctuary et al., 2019; Żebrowska et al., 2021). Moreover, the presence of GI symptoms correlates with the apparent behavioral manifestations in ASD children such as anxiety, self-injury, and aggression, indicating that exacerbated autism symptoms are explained partially by the underlying GI problems (Neuhaus et al., 2018). There is growing scientific evidence that GI comorbidity may have secondary effects on problematic behaviors of ASD individuals (Lasheras et al., 2020). Meanwhile, significant alterations in the stability, diversity, composition of the gut microbiota, and several microbial metabolic pathways were apparently detected in ASD children (Ho et al., 2020). Disrupted intestinal permeability and evidence of a systemic and intestinal inflammation were also detected in ASD subjects (Riccio and Rossano, 2019; Yang et al., 2020).

It is widely accepted that antibiotics affect the composition and diversity of the gut microbiota (Angelucci et al., 2019). This is supported by the evidence that early exposure to antibiotics may result in long-term disturbances in the gut microbiota of ASD children, involved in the rising recurring GI symptoms and changes in the microbiota composition (Vargason et al., 2019). More recently, it was revealed that transplantation of the fecal sample extract from ASD children into pregnant rats caused typical behavioral characteristics of ASD in offspring, recapitulating the abnormal gut microbiota of ASD (Qi et al., 2021). Altogether, these data from animal and clinical studies suggested that abnormal gut microbiota could contribute to core and associated ASD symptoms.

Gut dysbiosis refers to shifts in composition and activity of the gut microbiota, leading to detrimental effects on host health (Shanahan et al., 2021). Gut dysbiosis could be induced by genetics, environmental factors, diet, disease, stress, and age, which might destroy the intestinal mucosal barrier and cause amyloid and lipopolysaccharide (LPS) leak, then further increase microbial molecules in blood and activate the hypothalamic-pituitary-adrenal axis; thereby triggering a systemic and CNS inflammation with disruption of the blood-brain barrier (Cristofori et al., 2021). An increasing amount of evidence has established a close relationship between gut microbiota dysbiosis and GI symptoms in ASD children (Wang et al., 2011; Gorrindo et al., 2012). Intriguingly, gut dysbiosis also directly lead to ASD-like behavior (Vuong and Hsiao, 2017). Thinking along these lines, in ASD individuals, microbiota dysbiosis promotes toxins and bacterial products to enter into the bloodstream via damaged intestinal barrier. The brain, in turn, modulates gut peristalsis and 
TABLE 1 | Altered gut microbiota composition in ASD animal models $(\uparrow=$ increased, $\downarrow=$ decreased $)$.

\begin{tabular}{|c|c|c|}
\hline Animal models & Findings & References \\
\hline \multicolumn{3}{|c|}{ Gut dysbiosis in ASD animal models induced by environmental risk factors } \\
\hline VPA-exposed Sprague-Dawley rats & $\begin{array}{l}\alpha \text {-Proteobacteria } \uparrow, \text { Eubateriaceae } \uparrow, \text { Enterobacteriaceae } \downarrow \text {, Rikenellaceae } \uparrow, \text { Staphylococcaceae } \uparrow, \\
\text { Anaerotruncus } \downarrow \text {, Anaerofustis } \uparrow, \text { Proteus } \uparrow, \text { Staphylococcus } \uparrow, \text { Allobaculum } \uparrow, \text { Males: } \\
\text { Bacteroidetes } \uparrow, \text { Bacteroidia } \uparrow, \alpha \text {-Proteobacteria } \uparrow, \text { Females: Actinobacteria } \uparrow, \text { Allobaculum } \uparrow, \\
\text { Odoribacter } \uparrow, \text { Staphylococcus } \uparrow, \text { Bifidobacterium } \uparrow, \text { and Candidatus Arthromitus } \uparrow\end{array}$ & Liu et al. (2018) \\
\hline VPA-exposed male Wistar rats & $\begin{array}{l}\text { Fourth week: Prevotellaceae } \uparrow, \text { Streptococcaceae } \uparrow \text {, Desulfovibrionaceae } \uparrow, \text { Ruminiclostridium } 6 \downarrow \text {, } \\
\text { Lactobacillus } \downarrow \text {, Ruminococcaceae UCG-010 } \downarrow \text {, Ruminococcaceae UCG-004 } \downarrow \text {, and Candidatus } \\
\text { Arthromitus } \downarrow \text {, Eighth week: Marvinbryantia } \downarrow \text { and Helicobacter } \downarrow\end{array}$ & Kong et al. (2021a) \\
\hline $\begin{array}{l}\text { VPA-exposed male and female BALB/ } \\
\text { C mice }\end{array}$ & $\begin{array}{l}\text { Bacteroidales } \downarrow \text {, Deltaproteobacteria } \downarrow \text {, Clostridiales } \uparrow \text {, and Erysipelotrichales } \uparrow \text {, Males: Alistipes } \uparrow \text {, } \\
\text { Enterorhabdus } \uparrow \text {, Erysipelotrichalis } \uparrow, \text { Lactobacillales } \uparrow \text {, and Mollicutes } \uparrow\end{array}$ & de Theije et al. (2014) \\
\hline MIA mouse model & $\begin{array}{l}\text { Porphyromonadaceae } \uparrow \text {, Erysipelotrichaceae } \downarrow \text {, Alcaligenaceae } \downarrow \text {, Prevotellaceae } \uparrow \text {, } \\
\text { Ruminococcaceae } \downarrow \text {, unclassified Bacteriodales } \uparrow \text {, and Lachnospiriceae } \uparrow\end{array}$ & Hsiao et al. (2013) \\
\hline MHFD mouse model & $\begin{array}{l}\text { Lactobacillus reuteri } \downarrow \text {, Bifidobacterium pseudolongum } \downarrow \text {, Parabacteroides distasonis } \downarrow \text {, Bacteroides } \\
\text { uniformis } \downarrow \text {, Olsenella unclassified } \downarrow \text {, Collinsella unclassified } \downarrow \text {, Lactobacillus johnsonii } \downarrow \text {, and } \\
\text { Helicobacter hepaticus } \downarrow\end{array}$ & Buffington et al. (2016) \\
\hline p-Cresol-exposed mice & $\begin{array}{l}\text { Duncaniella dubosii } \uparrow, \text { Barnesiella sp. } \uparrow, \text { Anaerobium sp. } \uparrow, \text { Muribaculaceae bacterium } \uparrow, \text { Turicimonas } \\
\text { muris } \uparrow, \text { Eisenbergiella sp. } \downarrow \text {, Lacrimispora saccharolytica } \downarrow \text {, Anaerobium sp. } \downarrow \text {, Clostridiaceae } \\
\text { bacterium } \downarrow \text {, and Ruthenibacterium lactatiformans } \downarrow\end{array}$ & $\begin{array}{l}\text { Bermudez-Martin et al. } \\
\text { (2021) }\end{array}$ \\
\hline \multicolumn{3}{|c|}{ Gut dysbiosis in BTBR mouse model of idiopathic autism } \\
\hline BTBR mice & Clostridium cluster XI $\uparrow$, Bacteroidetes $\uparrow$, and Firmicutes $\downarrow$ & Newell et al. (2016) \\
\hline BTBR mice (12 months of age) & $\begin{array}{l}\text { Dehalobacterium } \downarrow \text {, Bacteroides } \uparrow, \text { Parabacteroides } \uparrow, \text { Females: Prevotella } \uparrow, \text { Coprobacillus } \uparrow, \\
\text { Sutterella } \uparrow, \text { Akkermansia (muciniphila) } \uparrow, \text { Oscillospira } \downarrow \text {, Males: Lactobacillus } \uparrow, \text { Ruminococcus } \downarrow \text {, } \\
\text { unclassified member of Helicobacteriaceae } \uparrow \text {, and Desulfovibrio } \downarrow\end{array}$ & Coretti et al. (2017) \\
\hline Adult BTBR male mice & $\begin{array}{l}\text { Akkermansia } \uparrow, \text { Bacteroides } \uparrow, \text { Bilophila } \uparrow, \text { Desulfovibrio } \downarrow \text {, Bifidobacterium } \downarrow \text {, Rikenella } \downarrow \text {, Blautia } \downarrow \text {, } \\
\text { Odoribacter } \downarrow \text {, and Parabacteroides } \downarrow\end{array}$ & Golubeva et al. (2017) \\
\hline \multicolumn{3}{|c|}{ Gut dysbiosis in monogenetic mutation mouse models of autism } \\
\hline Shank3 KO mice & $\begin{array}{l}\text { Lactobacillale } \downarrow \text {, Lactobacillaceae } \downarrow \text {, Veillonellaceae } \uparrow \text {, Bacteroides } \downarrow \text {, Lactobacillus } \downarrow \text {, Bacilli } \downarrow \text {, } \\
\text { Veillonella } \uparrow, \text { Coprococcus } \downarrow \text {, Prevotella } \downarrow \text {, Acetobacter } \downarrow \text {, and Turicibacter } \downarrow\end{array}$ & Tabouy et al. (2018) \\
\hline NL3 ${ }^{\mathrm{R} 451 \mathrm{C}}$ mice & Firmicutes $\uparrow$, Clostridia $\uparrow$, and Candidate $\downarrow$ & Hosie et al. (2019) \\
\hline Fmr1 KO2 mice & $\begin{array}{l}\text { Allobaculum } \uparrow, \text { Akkermansia } \uparrow, \text { Sutterella } \uparrow, \text { Odoribacter } \uparrow, \text { Desulfovibrio } \uparrow, \text { Turicibacter } \uparrow, \\
\text { Bifidobacterium } \uparrow, \text { Flexispira } \downarrow \text {, Bacteroides } \downarrow \text {, and Oscillospira } \downarrow\end{array}$ & Altimiras et al. (2021) \\
\hline $15 q$ dup mice & Species diversity of the microbiome $\downarrow$; number of OTUs $\downarrow$ & Septyaningtrias et al. (2020) \\
\hline EphB6 KO mice & Deferribacteres $\downarrow$ and Mucispirillum $\downarrow$ & Li et al. (2020) \\
\hline
\end{tabular}

EphB6 KO mice

Deferribacteres $\downarrow$ and Mucispirillum $\downarrow$

Li et al. (2020)

sensory and secretion functions, linking sympathetic glutamatergic neurons in the CNS to the gut through the vagus nerve (Zhu et al., 2018). Thus, inflammation and local damage in the brain also leads to gut dysbiosis, which become a promising therapeutic target. Likewise, we have recently reported that traumatic brain injury in mice induced significant gut dysbiosis such as increasing enrichment of the class Alphaproteobacteria and the families Porphyromonadaceae and Pseudomonadaceae, which are associated with neuroinflammation, brain edema, neuronal loss, and sensorimotor deficits (Ma et al., 2019). Lactobacillus acidophilus treatment resulted in the remodeling of the gut microbiota and rescuing CNS dysfunction (Ma et al., 2019).

\section{GUT DYSBIOSIS IN ASD ANIMAL MODELS AND PATIENTS}

\subsection{Gut Dysbiosis in ASD Animal Models}

Animal studies have also indicated that gut microbiota alterations lead to profound changes in ASD-like behaviors. The composition of gut microbiota in rodents showing features of ASD was detected in models of environmental risk factors such as valproic acid (VPA) exposure, maternal immune activation (MIA), maternal high-fat diet (MHFD) and p-Cresol exposure, idiopathic model for autism (BTBR mice), and monogenetic mutation mouse models of autism such as Shank3 KO mice, NLGN3 ${ }^{\text {R451C }}$ mutants, and EphB6 KO mice (Hsiao et al., 2013; Buffington et al., 2016; Newell et al., 2016; Liu et al., 2018; Tabouy et al., 2018; Cai et al., 2019; Hosie et al., 2019; Li et al., 2020; Bermudez-Martin et al., 2021; Zhong et al., 2020). The altered gut microbiota composition in ASD animal models is summarized in Table 1.

\subsection{Gut Dysbiosis in ASD Animal Models Induced by Environmental Risk Factors}

Mice exposed to VPA in utero exhibited altered gut microbiota composition and presented with a decrease of Bacteroids, Deltaproteobacteris, and Erysipelotrichales as well as an increase of Clostridiales (de Theije et al., 2014). An impact of gender on the gut microbiota composition of VPA in utero- 
exposed mouse offspring was observed, characterized by increased levels of Alistipes, Enterorhabdus, Mollicutes, Lactobacillales, and Erysipelotrichalis in males (de Theije et al., 2014). Liu et al. (2018) have confirmed that sex-specific differences in gut microbiota composition also existed in VPA exposed rats. VPA-exposed rats displayed decreased microbial diversity and increased in the abundance of $\alpha$-Proteobacteria, Eubateriaceae, Rikenellaceand, and Staphylococcaceae. At the genus level, VPA exposure increased the abundance of the genera Allobaculum, Anaerofustis, Proteus, and Staphylococcus significantly (Liu et al., 2018). One recent study has further reported that there was a significant difference in the composition of gut microbiota and SCFA levels between autistic-like and healthy rats during weaning and sexual maturation (Kong et al., 2021a). Evidence has indicated that the MIA offspring display damaged GI integrity, microbiota dysbiosis, and changes in serum metabolites that are similar to ASD endophenotypes (Hsiao et al., 2013). Principal coordinate analysis (PCoA) showed robust differences in the diversity of Clostridia and Bacteroidia between MIA offspring and controls. MIA offspring displayed increased abundance in the families of Lachnospiraceae, Porphyromonadaceae, and Prevotellaceae, although the species richness was not altered. Moreover, specific Lachnospiraceae, along with other Bacteroidial species, might be involved in the pathogenesis of MIA-induced autistic behavior and impact MIA-associated GI abnormalities (Hsiao et al., 2013). In the MHFD mouse model for autism, the diversity of microbiota was lower than that in the control mice, with significant reductions in Lactobacillus, Parabacteroides, Helicobacter, and B. Uniformis (Buffington et al., 2016). Mice exposed to the microbial metabolite p-Cresol for 4 weeks in drinking water exhibited social interaction deficits and stereotypies, reminiscent of ASD core symptoms in humans (Bermudez-Martin et al., 2021). Moreover, the microbiota from p-Cresol-treated mice induced social behavior deficits and significant divergences in microbial composition when transplanted to untreated recipients (Bermudez-Martin et al., 2021). Importantly, a growing body of independently replicated findings revealed that germ-free (GF) mice, devoid all microorganisms in or on their bodies, displayed impaired social behavior, which indicated that the absence of bacterial colonization during development exerts a detrimental effect on animal social behavior (Stilling et al., 2015; Stilling et al., 2018). Additionally, it has been further supported by a study that the GF mice colonized with microbiota from children with ASD promoted ASD-relevant behaviors in GF mice and affected the distribution and composition of intestinal microorganism (Xiao et al., 2021).

\subsection{Gut Dysbiosis in BTBR Mouse Model of Idiopathic Autism}

Noteworthy, BTBR mice displayed a global alteration of microbial communities of cecal and fecal samples, suggesting that this model may be useful to interpret gut-brain interactions in ASD (Newell et al., 2016; Coretti et al., 2017; Golubeva et al., 2017). Measurement of 16S rRNA sequences showed BTBR mice harbored a different fecal microbial community in gut such as increased Clostridium cluster XI, Bacteroidetes, and decreased Firmicutes (Newell et al., 2016). Moreover, BTBR mice fed a chow diet showed a significantly increased level of A. muciniphila, the predominant representative of the Verrucomicrobia phylum within the GI tract in both cecal and fecal matter, which are linked to maintaining homeostasis of mucus secretions (Newell et al., 2016). In particular, Bacteroides, Parabacteroides, Sutterella, Dehalobacterium, and Oscillospira genera as key drivers of sexspecific gut microbiota composition have been identified in BTBR mice (Coretti et al., 2017). The genera Bacteroides and Oscillospira were indicated to be related to pathological traits, which might help to understand the sex-induced alteration of behavior, gut integrity, and colon immunological state in BTBR mice (Coretti et al., 2017). Similarly, Golubeva et al. (2017) showed a substantially reduced gut bacterial diversity in the BTBR cecum. PCoA revealed that BTBR mice displayed an increase in Bacteroidetes and a decrease in Firmicutes at the phylum level; an increase in Akkermansia, Bacteroides, and Bilophila genera; and a reduction in Bifidobacterium and Desulfovibrio (Golubeva et al., 2017).

\subsection{Gut Dysbiosis in Monogenetic Mutation Mouse Models of Autism}

In the case of autism, studies have shown autism-associated genes also influence the microbiome (Tabouy et al., 2018; Hosie et al., 2019; Liu et al., 2021). Using the LefSe bioinformatic tool, Tabouy et al. (2018) found that the bacterial richness was decreased in the Shank3 KO mice, with a decrease in the relative abundance of members of the class Bacilli, order Lactobacillales, family Lactobacillaceae, and genus Lactobacillus. At the genus level, Coprococcus, Bacteroides, Acetobacter, Turicibacter, and Prevotella were also decreased in the Shank3 KO mice, while the family Veillonellaceae and genus Veillonella were increased in the Shank3 KO mice (Tabouy et al., 2018). Neuroligin 3 (NLGN3) has been confirmed as risk alleles for ASD (Jamain et al., 2003). Interestingly, NLGN3 KO mice displayed gut dysfunction such as decreased colonic smooth muscle tone and altered colonic motility. Furthermore, NLGN3 $3^{\mathrm{R} 451 \mathrm{C}}$ mutants showed altered fecal microbial communities including increased three operational taxonomic units (OTUs) belonging to the phylum Firmicutes, class Clostridia, as well as one decreased OTU from the Candidate phyla (Hosie et al., 2019). Fragile X syndrome (FXS) is considered as the leading monogenetic cause of autism, and the FXS phenotype could be well recapitulated in Fmr1 KO2 mice. Using $16 \mathrm{~S}$ ribosomal RNA gene sequencing, Altimiras et al. (2021) identified bacterial species alterations in the gut microbiome of Fmr1 KO2 mice including an increase in the Firmicutes, Bacteroides, and Verrucomicrobia phyla and Sutterella and Akkermansia genera and a decrease in the Prevotella genus. Septyaningtrias et al. (2020) also found a significant reduction in species diversity of the microbiome and OTU number in fecal samples in a mouse model for ASD with the human 15q11-13 duplication ( $15 q d u p)$. EphB6, located on chromosome $7 \mathrm{q}$, is identified as a candidate ASD-associated gene. One report has indicated that EphB6 KO mice displayed altered gut microbial composition in the fecal microbiota including a decreased 
TABLE 2 | Altered gut microbiota composition in ASD patients ( $\uparrow=$ increased, $\downarrow=$ decreased).

Study subjects

13 autistic children and 8 control children

15 autistic children and 8 control children 58 autistic children, 12 healthy siblings, and 10 control children

33 autistic subjects, 7 sibling controls, and 8 nonsibling control subjects

15AUT-GI and 7 control-Gl children

10 autistic children, 9 siblings, and 10 healthy children

35 children with ASD and 6 TD children

48 children with ASD and 48 healthy children

77 children with ASD and 50 age-matched healthy children

9 autistic children, and 6 healthy children

143 ASD children, 143 age- and sex-matched TD individuals

72 ASD and 74 TD children

11 ASD and 14 healthy control children
Findings

References

Clostridium $\uparrow$ and Ruminococcus spp. $\uparrow$

C. bolteae $\uparrow$, Clostridium clusters $1 \uparrow$, and Clostridium clusters XI Clostridium clusters $\mathrm{I}$ and Clostridium clusters $\| \uparrow$

Bacteroidetes $\uparrow$, Proteobacteria $\uparrow$, Alkaliflexus $\uparrow$, Desulfovibrio $\uparrow$, Acetanaerobacterium $\uparrow$, Bacteroides $\uparrow$, Parabacteroides $\uparrow$, Desulfovibrio spp. $\uparrow$, Bacteroides vulgatus $\uparrow$, Actinobacteira $\downarrow$, Turicibacter Clostridium $\downarrow$, Firmicutes $\downarrow$, Weissella $\downarrow$, Helcococcus $\downarrow$, Alkaliphilus $\downarrow$ Anaerofilum $\downarrow$, Pseudoramibacter $\downarrow$, Ruminococcus $\downarrow$, Streptococcus $\downarrow$, Anaerovorax $\downarrow$, Dialister $\downarrow$, Lactococcus $\downarrow$, Leuconostoc $\downarrow$, and Ethanoligenens $\downarrow$

Betaproteobacteria $\uparrow$, Bacteroidetes $\downarrow$, and the ratio of Firmicutes/Bacteroidetes $\uparrow$

Lactobacillus spp. $\uparrow$, Desulfovibrio spp. $\uparrow$, and Bacteroidetes/Firmicutes $\downarrow$

Sutterella $\uparrow$, Odoribacter $\uparrow$, Butyricimonas $\uparrow$, Veillonella $\downarrow$, Streptococcus $\downarrow$, and Bacteroidetes/ Firmicutes $\uparrow$

Firmicutes $\downarrow$, Proteobacteria $\downarrow$, Verrucomicrobia $\downarrow$, Bacteroidetes/Firmicutes $\uparrow$, Dialister $\downarrow$, Prevotella $\uparrow$, Bacteroides $\uparrow$, Megamonas $\uparrow$, Escherichia/Shigella $\downarrow$,

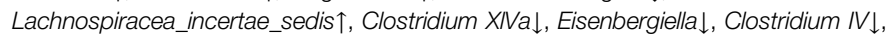
Flavonifractor $\downarrow$, Haemophilus $\downarrow$, and Akkermansia $\downarrow$

Unidentified Lachnospiraceae $\uparrow$, Clostridiales $\uparrow$, Dorea $\uparrow$, Erysipelotrichaceae $\uparrow$, Collinsella $\uparrow$, Lachnoclostridium $\uparrow$, Bacteroides $\downarrow$, Faecalibacterium $\downarrow$, Parasutterella $\downarrow$, and Paraprevotella $\downarrow$ Bacteroidales $\downarrow$, Selenomonadales $\downarrow$, Prevotellaceae $\downarrow$, and Ruminococcaceae $\uparrow$

Firmicutes $\uparrow$, Firmicutes/Bacteroidetes $\uparrow$, Megamonas $\downarrow$, Proteobacteria $\uparrow$, Actinobacteria $\uparrow$, Bacteroidetes $\downarrow$, Dialister $\uparrow$, Escherichia-Shigella $\uparrow$, Bifidobacterium $\uparrow$, Prevotella $9 \downarrow$, and

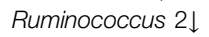

Clostridium $\uparrow$, Dialister $\uparrow$, Coprobacillus $\uparrow$, and Faecalibacterium $\downarrow$

Actinobacteria $\downarrow$, Bacteroidetes $\uparrow$, Proteobacteria $\uparrow$, Bacteroidetes/Firmicutes $\uparrow$,

Finegold et al.

(2002)

Song et al. (2004)

Parracho et al.

(2005)

Finegold et al.

(2010)

Williams et al.

(2011)

Tomova et al. (2015)

Zhang et al. (2018)

Zou et al. (2020)

Ding et al. (2020)

Sun et al. (2019)

Dan et al. (2020)

Wan et al. (2021) Coretti et al. (2018) Actinomycetaceae $\downarrow$, Coriobacteriaceae $\downarrow$, Oscillospira $\uparrow$, Gemellaceae $\downarrow$, Streptococcaceae $\downarrow$, Faecalibacterium prausnitzii $\uparrow$, and Bifidobacteriaceae $\downarrow$

abundance of the phylum Deferribacteres, especially Mucispirillum at the genus level (Li et al., 2020).

\subsection{Gut Dysbiosis in ASD Patients}

It has been noted that the gut microbiota stabilization occurs around the age of 2 to 3 , a critical period for the ASD onset (Rautava et al., 2012). GI system disorder is a common comorbidity in children with ASD. Disruptions in the gut bacteria profiles may tend to strongly correlate with the increased risk and severity of autism (Zhang et al., 2018). It is supposed that a neurotoxin produced by bacteria reaches the brain via the vagus nerve and may lead to communication and social impairments. The altered gut microbiota composition in ASD patients is summarized in Table 2.

The finding of a microbial element involved in autism pathogenesis was reported in 1998 (Bolte, 1998). Several pieces of evidence have shown that the compositional changes in the gut microbiota are related to alterations in the normal function of the nervous system and behavioral deficits in ASD (Williams et al., 2011; Ding et al., 2020; Ho et al., 2020). Finegold et al. (2002) found differences in Clostridial species between children with late-onset autism and control children. Typically, non-sporeforming anaerobes and microaerophilic bacteria were in the intestinal flora of ASD children but absent in control children (Finegold et al., 2002). Consistent with this finding, Song et al. (2004) revealed that Clostridial groups such as Clostridium bolteae and clusters I and XI in feces significantly elevated in
ASD children. Parracho et al. (2005) found the Clostridium histolyticum group (Clostridium clusters I and II) abundant in the fecal flora of ASD children.

The investigation of the dominant intestinal bacterial phyla indicates that the dysbiosis exhibits a similar trend, primarily characterized by the alterations in the ratio and the composition of the primary bacterial phyla (Firmicutes, Bacteroidetes, Fusobacteria, and Verrucomicrobia) (De Angelis et al., 2015). Finegold et al. (2010) found that the phylum level of Bacteroidetes and Proteobacteria; the genus level of Alkaliflexus, Desulfovibrio, Acetanaerobacterium, Parabacteroides, and Bacteroides; and the species of Desulfovibrio spp. and Bacteroides vulgatus were higher in the ASD group than those in controls. Also, from the phylum level, Actinobacteira and Firmicutes and from the genus level Weissella, Turicibacter, Clostridium, Anaerofilum, Pseudoramibacter, Ruminococcus, Streptococcus, Anaerovorax, Dialister, Lactococcus, Leuconostoc, Ethanoligenens, Helcococcus, and Alkaliphilus were higher in the control group than those in the ASD group (Finegold et al., 2010). One study in a cohort of young ASD children (2-4 years of age) and age-matched neurotypical healthy controls has indicated that ASD children displayed a significant increase in Bacteroidetes and Proteobacteria and a decrease in Actinobacteria (Coretti et al., 2018). Of note, Bifidobacterium longum, the dominant bacterium in infant gut microbiota, was significantly depleted, while Faecalibacterium prausnitzii, a late colonizer of healthy human gut and major butyrate producer, 


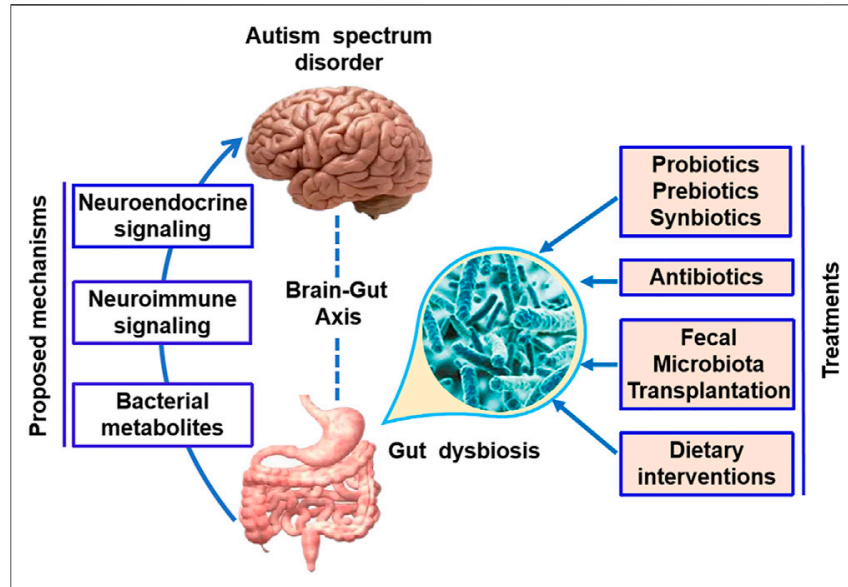

FIGURE 1 | Microbial-based therapeutic interventions in ASD. The gut microbiota has been found to affect brain function through the neuroendocrine signaling, neuroimmune signaling, and bacterial metabolites. Potential microbial-based therapeutic interventions in ASD include prebiotic/ probiotic/synbiotic, antibiotics, fecal microbiota transplantation, and dietary interventions.

was increased in ASD patients (Coretti et al., 2018). Likewise, one recent study reported that the genus level of Bacteroides, Prevotella, Lachnospiracea_incertae_sedis, and Megamonas as well as Bacteroidetes/Firmicutes increased in autistic children relative to the control children (Zou et al., 2020).

Differently, another study in ASD child cohort has confirmed that gut dysbiosis is characterized by a decrease in Bacteroidetes and ratio of Bacteroidetes to Firmicutes and a greater preponderance of Betaproteobacteria in the intestinal biopsy samples (Williams et al., 2011). Tomova et al. (2015) have found a significantly decreased Bacteroidetes/Firmicutes ratio, but increased counts of Lactobacillus spp., detected in ASD children in Slovakia. They also noticed that a trend of increase in Delsulfovibrio spp. was especially strongly associated with the severity of autism. Sun et al. (2019) have found a large difference in the abundance of microbiota at the level of family, genus, and species between the ASD group and the healthy control group. Ding et al. (2020) have shown that gut microbiota in ASD children displayed higher biomass, richness, and biodiversity and an altered microbial community structure at the genus level in the ASD group. Notably, three altered intestinal microbiome strains in ASD children, namely, Erysipelotrichaceae, Faecalibacterium, and Lachnospiraceae, were related to the production of butyric acid in the gut and were positively correlated with ASD severity (Ding et al., 2020).

As stated, numerous studies have demonstrated the variation in the gut microbiota of autistic children (Dan et al., 2020; Wan et al., 2021), but there is little consensus on the uniform microbial profile of individuals with ASD and disparities in currently available data. Heterogeneity in population studied, sample collection, and study design all result in variability in microbiome studies. This may be caused by methodological dissimilarities and the differences between the studied objects. Moreover, age is a significant factor leading to this deviation.
Patients at different ages have some differences in gut microbiota. In younger children, gut microbiota remains unstable (Rautava et al., 2012). Thus, ongoing research is further needed to identify the association between microbiota alterations and ASD.

\subsection{Pathogenesis Mechanisms Underlying Gut Dysbiosis Involved in the ASD}

A variety of studies have indicated that the gut microbiota affects brain function through neuroendocrine signaling, neuroimmune signaling, and bacterial metabolites (Figure 1) (Li et al., 2017).

\subsection{Neuroendocrine Signaling}

The enteric nervous system (ENS) consisting of millions of neurons regulating GI functions is referred to as a "second brain" (Zhang et al., 2014). The ENS may interplay with the intestinal bacteria directly or indirectly. The intestinal microbes affect the biologically active peptides and neuroactive molecules in the gut and blood. Besides, intestinal bacteria play critical roles in the production of neurotransmitters in the intestinal lumen through host biosynthetic pathways, which might be the molecular basis for the alterations in neurotransmitter metabolism (O'Donnell et al., 2020). It has been confirmed that Lactobacillus spp. is capable of producing $\gamma$-aminobutyric acid (GABA) and acetylcholine. Similarly, Bacillus spp. and Serratia spp. have been found to produce dopamine, while Candida spp., Streptococcus spp., Escherichia spp., and Enterococcus spp. produce serotonin (5-hydroxytryptamine, 5HT), a particularly important brain neurotransmitter, deeply affecting intestinal physiology and the behavior. In support of this, evidence suggests that the microbiota can affect dopamine circuits and vagal sensory neurons in the gut, which is essential for central dopamine function (Travagli et al., 2020). Moreover, Reigstad et al. (2015) also found that the gut microbiota acts through SCFAs, which have the ability to promote the enteric 5HT production and homeostasis. These produced neurotransmitters can transduce signals to the CNS, then modulate the behavior via acting on different neural circuits. The increase in Clostridales, along with a decrease in Dorea, Blautia, and Sutterella, could also alter host processing of tryptophan, the precursor of serotonin, and an overabundance of serotonin (Luna et al., 2017). This in turn apparently caused an increase in the intestinal serotonergic effects and serotonin deficiency in the host, which may be associated with deficits in mood and cognitive function seen in ASD (Luna et al., 2017).

\subsection{Neuroimmune Signaling}

It has been suggested that the gut can also interact with the brain through immunological pathways, and the altered host immune responses arising from gut microbiota are closely related to ASDrelevant symptoms (Ashwood et al., 2006; Cristiano et al., 2018). The bacterial cell wall constituents persistently stimulate the innate immune system to produce inflammatory cytokines, consequently keeping a basal state of immune activation at the intestinal mucosal surface and influencing the whole body (Duerkop et al., 2009). Immune responses to toxins generated 
by pathogenic microbiota and focal inflammation induce an increase in gut permeability. The defects in the gut barrier further cause the toxins and bacterial products to enter into the bloodstream, which might result in brain dysfunction (Yoo et al., 2020).

Luna et al. (2017) found that an increase in Clostridales, combined with a decrease in Dorea, Blautia, and Sutterella, could change pro-inflammatory cytokines, which can reach the brain through blood flow. Bacteroides fragilis and several members of the genus Clostridia mediate an anti-inflammatory action through promoting the production of anti-inflammatory cytokines, such as IL-10 and IL-13, whereas some pathogenic bacteria (Salmonella typhimurium and C. difficile) trigger the production of inflammatory cytokines (Lombardi et al., 2018). Hydrogen sulfide, which is generated by bacteria such as Prevotella during anaerobic respiration, is related to increased intestinal inflammation (Yano et al., 2015). Cao et al. (2021) have shown a positive correlation between plasma IFN- $\gamma$ level and Pseudomonas, Streptomyces, and Clostridium relative abundances, whereas a negative correlation between plasma IFN- $\gamma$ level and Blautia relative abundance. Furthermore, a positive correlation between plasma IL- 6 concentration and relative abundance of Bacillus was also observed. Besides, they found butyrate-producing bacteria, such as Anaerostipes and Coprococcus, were negatively correlated with IL-6 in severe ASD subjects (Cao et al., 2021). LPS, a major component of the cell wall of gram-negative bacteria, can induce the immune response of mammalian cells via lipid A, its toxic component. The data of Maigoro and Lee (2021) showed a significant increase in the prevalence of gram-negative bacteria in the gut of individuals with ASD compared to healthy subjects. Moreover, LPS could also be produced by gut microbiota, absorbed into the blood across an injured gut wall, and activate Toll-like receptors in the ENS and CNS (Al-Asmakh and Hedin, 2015; Garcia- Gutierrez et al., 2020). Non-canonical NLRP3 inflammasome activation by gram-negative bacteria (i.e., Citrobacter rodentium, Escherichia coli, Legionella pneumophila, Salmonella typhimurium, and Vibrio cholerae) further activated the transcription of IL- $1 \beta$, IL-18, and NLRP3 through NF- $\kappa \mathrm{B}$ activation (Pellegrini et al., 2020).

\subsection{Bacterial Metabolites}

There is growing evidence revealing that certain metabolites generated by gut microbes are responsible for ASD. Liu et al. (2021) found that the single-nucleotide variations in ASD were significantly enriched in genes correlated with the microbiome composition and a broad aspect of microbial functions, especially metabolism. Disordered compounds such as SCFAs, p-Cresol, dimethylamine, hippuric acid, and phenylacetylglutamine were detected in patients with ASD. These compounds are widely derived from gut bacteria, which induce autistic behavior by influencing and regulating the host's immune and nervous systems. Generally, the increase of toxic metabolism and decrease of protective metabolism are observed in gut dysbiosis (Sivamaruthi et al., 2020). Several pieces of evidence have indicated that a lower abundance of protective bacteria plays critical roles in decreasing oxidative stress, cell detoxification, and excretion of heavy metal (Engwa et al., 2016; Khatua et al., 2017).

With an increasing abundance of toxin-forming bacteria in ASD children, some metabolites derived from the activity of detrimental bacteria are closely related to the ASD pathogenesis. Clostridiaceae is regarded to synthesize certain toxic metabolic products to humans, such as phenols, $\mathrm{p}$-Cresol, and certain indole derivatives (Dawson et al., 2008). One study has confirmed that the increased Clostridiales is closely correlated with repetitive behaviors and GI problems in ASD, which can be rescued by using antibiotic (Finegold, 2008). In ASD patients, increased relative abundance of Bacteroidetes is correlative with the level of SCFAs. Macfabe (2012) have found that propionic acid or other SCFAs can lead to the biological, chemical, and pathological changes that are features of autism.

\section{NOVEL THERAPEUTIC APPROACHES TARGETING GUT MICROBIOME FOR ASD}

\subsection{Probiotics/Prebiotics/Synbiotics}

Probiotics are live microorganisms that, when ingested or applied locally in sufficient amounts, can correct the dysbiosis. Bifidobacterium and Lactobacillus strains as well as Saccharomyces boulardii are commonly used as probiotics. Prebiotics are non-digestible components of the human body that benefit the host health by selectively stimulating the growth and activity of gut bacteria, particularly Lactobacillus and Bifidobacterium. Prebiotics, specifically galacto-oligosaccharides (GOSs), have also shown a beneficial impact on ASD symptoms and comorbidities. Synbiotics refers to a combination of both prebiotics and probiotics (Janmohammadi et al., 2021). Rebuilding the microbiome in the gut appears to be the promising therapeutic intervention in ASD.

\subsubsection{Treatment With Probiotics/Prebiotics/Synbiotics in the ASD Animals}

A plethora of studies on the role of probiotics in inflammation have been performed in ASD animals (Cristofori et al., 2021). One study has reported that orally given human commensal Bacteroides fragilis NCTC 9343 to MIA offspring effectively corrects gut permeability and microbial composition specified in diversity of Clostridia and Bacteroidia OTUs and improves ASD-like deficits (Hsiao et al., 2013). Wang et al. (2019) have further found that oral probiotics during pregnancy decreased the ASD-like behaviors induced by MIA in offspring and rescued parvalbumin positive neuron loss as well as the reduction of GABA in the prefrontal cortex of adult offspring. Consistently, one independent study also found that feeding with a probiotic Lactobacillus strain (L. plantarum L168) partially rescued the social behavior in $k d m 5$-deficient flies (Chen et al., 2019). The underlying mechanism is linked to transcriptionally regulate component genes related to the immune deficiency signaling pathway, which in turn keeps host-commensal bacteria homeostasis in a demethylase-dependent manner (Chen et al., 2019). Prebiotic (propolis and bee pollen) supplements have the 
TABLE 3 | Microbial-based therapeutic interventions in ASD patients.

\begin{tabular}{|c|c|c|c|}
\hline Type of trial & Treatments & Findings & References \\
\hline \multicolumn{4}{|c|}{ Treatments with probiotics/prebiotics/synbiotics in ASD patients } \\
\hline Case study & S. boulardii & $\begin{array}{l}\text { Reduced obsessive compulsive disorder and self-injurious } \\
\text { behavior }\end{array}$ & Kobliner et al. (2018) \\
\hline $\begin{array}{l}\text { Randomized, double-blind, } \\
\text { placebo-controlled pilot trial }\end{array}$ & $\begin{array}{l}\text { Lactobacillus plantarum PS128 + intranasal } \\
\text { oxytocin }\end{array}$ & $\begin{array}{l}\text { Improved ASD core socio-behavioral symptoms, clinical } \\
\text { global functioning, and gut microbiome dysbiosis }\end{array}$ & Kong et al. (2021c) \\
\hline $\begin{array}{l}\text { Randomized prospective } \\
\text { studies }\end{array}$ & Lactobacillus plantarumPS128 & $\begin{array}{l}\text { Increased attention, communication skills, and personal } \\
\text { autonomies }\end{array}$ & Mensi et al. (2021) \\
\hline $\begin{array}{l}\text { Randomized, double-blind, } \\
\text { placebo-controlled study }\end{array}$ & Lactobacillus plantarumPS128 & Ameliorated opposition/defiance behaviors & Liu et al. (2019) \\
\hline $\begin{array}{l}\text { A prospective, open-label } \\
\text { study }\end{array}$ & $\begin{array}{l}\text { Lactobacillus acidophilus, Lactobacillus } \\
\text { rhamnosus, and Bifidobacteria longum }\end{array}$ & $\begin{array}{l}\text { Have beneficial effects on both behavioral and Gl } \\
\text { manifestations }\end{array}$ & Shaaban et al. (2018) \\
\hline $\begin{array}{l}\text { Double-blind randomized, } \\
\text { placebo-controlled trial }\end{array}$ & Probiotics (De Simone Formulation) & $\begin{array}{l}\text { Improve core autism symptoms in the social-affective } \\
\text { domain }\end{array}$ & Santocchi et al. (2020) \\
\hline A randomized controlled trial & Probiotic mixture (Vivomixx ${ }^{\circledR}$ ) & Alleviated autistic symptoms & Santocchi et al. (2016) \\
\hline Case study & VSL\#3 & Improved autistic core symptoms and Gl symptoms & Grossi et al. (2016) \\
\hline $\begin{array}{l}\text { A randomized, double-blind, } \\
\text { placebo-controlled }\end{array}$ & $\mathrm{B}-\mathrm{GOS}^{\circledR}$ & $\begin{array}{l}\text { Improved anti-social behavior and significant increase of } \\
\text { Lachnospiraceae family }\end{array}$ & Grimaldi et al. (2018) \\
\hline $\begin{array}{l}\text { A double-blind, placebo- } \\
\text { controlled intervention study }\end{array}$ & Probiotics + FOS & Reduced the severity of autism and Gl symptoms & Wang et al. (2020b) \\
\hline $\begin{array}{l}\text { Randomized, double-blind, } \\
\text { crossover clinical trial }\end{array}$ & $\mathrm{BCP}+\mathrm{B}$. infantis & Reduced Gl symptoms and aberrant behaviors & Sanctuary et al. (2019) \\
\hline A placebo-controlled pilot trial & VISBIOME (eight probiotic species) & Improved parent-selected target symptoms & Arnold et al. (2019) \\
\hline Multi-center clinical study & $\begin{array}{l}\text { ABA training }+ \text { probiotics (six strains of } \\
\text { bacteria) }\end{array}$ & Decreased ATEC and Gl scores & Niu et al. (2019) \\
\hline Open-label study & Probiotic & Decreased levels of total SCFAs and lysozymes & Adams et al. (2011) \\
\hline Open-label trial & Lactobacillus acidophilus (strain Rosell-11) & Improved the ability to concentrate and fulfil orders & $\begin{array}{l}\text { Kałużna-Czaplińska and } \\
\text { Błaszczyk, (2012) }\end{array}$ \\
\hline
\end{tabular}

Treatments with antibiotics in the ASD patients

\begin{tabular}{|c|c|c|c|}
\hline Case report & Amoxicillin & $\begin{array}{l}\text { Improve speech, eye contact, and sleep behaviors and } \\
\text { reduced repetitive behaviors }\end{array}$ & Kuhn et al. (2012) \\
\hline Case reports & Vancomycin & $\begin{array}{l}\text { Ameliorated communication and several behavioral } \\
\text { defects }\end{array}$ & Sandler et al. (2020) \\
\hline
\end{tabular}

Treatments with FMT in the ASD patients

$\begin{array}{ll}\text { Open-label clinical trial } & \text { FMT } \\ \text { Open-label trial } & \text { FMT } \\ \begin{array}{l}\text { Open-label study } \\ \text { Follow-up study }\end{array} & \text { FMT } \\ \begin{array}{l}\text { Open-label, randomized } \\ \text { wait-list-controlled trial }\end{array} & \text { FMT }\end{array}$

Mitigated autism symptoms and Gl disorder;
reconstructed gut microbiota; and recovered the serum

Treatments with dietary interventions in the ASD patients

\begin{tabular}{|c|c|c|c|}
\hline $\begin{array}{l}\text { Parallel randomized double- } \\
\text { blind, placebo-controlled trial }\end{array}$ & Vitamin D & Alleviated CARS and ATEC scales & Javadfar et al. (2020) \\
\hline Randomized clinical trial & Gluten free diet & Improved gastrointestinal symptoms and ASD behaviors & Ghalichi et al. (2016) \\
\hline
\end{tabular}

potential in ameliorating neuroinflammation and dysbiosis in a rodent model of autism (Aabed et al., 2019).

One recent research interest in the neurotransmitter homeostasis is a link between brain and gut microbiota. Kong et al. (2021b) assayed the effect of three Lactobacillus strains ( $L$. helveticus CCFM1076, L. acidophilus La28, and L. acidophilus JCM 1132) on autistic-like behavioral symptoms in VPA-treated rats from weaning to sexual maturation. The authors reported that oral treatment of L. helveticus CCFM1076 for 4 weeks restored neurotransmitter homeostasis by improving the balance of the 5HT system in the PNS and CNS, thereby ameliorating autistic-like behaviors, while $L$. acidophilus La28 and L. acidophilus JCM 1132 did not (Kong et al., 2021b). One study was performed on 50 juvenile hamsters, in which the administration of a mixture of Bifidobacteria and Lactobacilli strains (ProtexinR) 
alleviated glutamate excitotoxicity through restoring the depleted GABA and $\mathrm{Mg}^{2+}$ as well as reducing glutamate (El-Ansary et al., 2018). This might explain the underlying mechanisms of probiotics involved in the improvement of autistic-like behaviors.

It is worth noting that bacterial species are sensitive to an autism-related mutation. Treatment of L. reuteri in Shank3 KO mice produced beneficial effects on social and repetitive behaviors, which is correlated with altered GABA receptor levels in multiple brain regions (Tabouy et al., 2018). Consistent with this study, Sgrittal et al. (2019) further confirmed that $L$. reuteri corrected social deficits in several tested ASD mouse models (MHFD, VPA, GF, and BTBR mice) through restoring the composition of the host's gut microbiota, regardless of the initial insult triggering the disorder. Instead, L. reuteri acts in a vagus nerve-dependent manner and promotes social interaction-induced long-lasting synaptic plasticity in the mesolimbic dopamine reward system of ASD mice (Sgritta et al., 2019). In addition, one recent study confirmed that probiotic treatment could reconstitute the gut microbiome composition in Dip2a KO mice (Zhang et al., 2021).

\subsubsection{Treatment With Probiotics/Prebiotics/Synbiotics in the ASD Patients}

Currently, several therapeutic trials have explored the efficacy of probiotics for treating ASD symptomatology (Table 3). In a randomized, double-blind, placebo-controlled trial, Lactobacillus plantarum PS128 (PS128) administered to boys with ASD aged 7-15 for 4 weeks ameliorated opposition/defiance behaviors (Liu et al., 2019). In line with this study, Mensi et al., 2021 found that autistic children and adolescents who received L. plantarum PS128 had greater improvements in terms of global functioning characterized by increased attention, communication skills, and personal autonomies. In another randomized, doubleblind, placebo-controlled pilot trial, individuals with ASD aged 3-20 years who received oral probiotic L. plantarum PS128 and intranasal oxytocin elicited significant improvements in ASD core socio-behavioral symptoms, clinical global functioning, and gut microbiome dysbiosis (Kong et al., 2021c). It was revealed that the two treatments are supposed to have synergistic interactions in the regulation of the gut-brain axis. A case study reported by Kobliner et al. (2018) revealed that the supplementation of $S$. boulardii, a non-pathogenic probiotic yeast, successfully reduced obsessive-compulsive disorder and self-injurious behavior in a 16-year-old ASD subject.

Likewise, one study in an Egyptian cohort of 30 autistic children indicates that 3 months of supplementation of three probiotic strains (L. acidophilus, Lactobacillus rhamnosus, and Bifidobacteria longum) have beneficial effects on both behavioral and GI manifestations of ASD as well as the colony counts of Bifidobacteria and Lactobacilli levels (Shaaban et al., 2018). In a double-blind, randomized, placebo-controlled trial, which was carried out on 85 preschoolers with ASD, probiotics (De Simone Formulation) containing 450 billion of eight probiotic strains was administered in 42 infants and a placebo in the remaining infants in the control group for 6 months; the outcome revealed that administration of probiotic may potentially improve core autism symptoms in the socialaffective domain in a subset of ASD children independent of the specific intermediation of the probiotic effect on GI symptoms (Santocchi et al., 2020). A randomized controlled trial from Santocchi et al. (2016) has reported that individuals with ASD and GI symptoms treated with a probiotic mixture $\left(\right.$ Vivomixx ${ }^{\circledR}$ ) showed dramatically alleviated autistic symptoms in behavioral profiles and in cognitive, linguistic, and adaptive functioning compared to placebo-treated individuals with ASD and GI symptoms. VSL\#3, a multistrain mixture of eight probiotics, administration in humans has been demonstrated to increase Lactobacilli and Bifidobacteria count (Rajkumar et al., 2014). One study from Grossi et al. (2016) revealed that both autistic core symptoms and GI symptoms in a boy (12 years old) with ASD and severe cognitive dysfunction were improved markedly by the supplementation of VSL\#3 for 4 weeks. A pilot crossover trial has confirmed that supplementation with VISBIOME formulation containing eight probiotic species in ASD children aged 3-12 displayed significant improvement in GI complaints compared with placebo treatment (Arnold et al., 2019).

Additionally, the combined therapies address multiple aspects of the ASD. A combined dietary approach including 6-week Bimuno $^{\circledR}$ galactooligosaccharide $\left(\mathrm{B}-\mathrm{GOS}^{\circledR}\right)$ prebiotic and exclusion diet intervention in 30 autistic children resulted in a significant increase of Lachnospiraceae family, as well as significant changes in fecal and urine metabolites, which are related to improvements in anti-social behavior (Grimaldi et al., 2018). Similarly, one recent study was conducted to evaluate the effect of combination treatment with probiotics (a mixture of four probiotic strains) and fructo-oligosaccharide (FOS) in ASD children. It was revealed that probiotics + FOS intervention increased beneficial bacteria (Bifidobacteriales and Bifidobacterium longum) and suppressed suspected pathogenic bacteria (Clostridium), with marked alleviation in the severity of autism and GI symptoms (Wang et al., 2020b). Bovine colostrum product (BCP), a source of prebiotic oligosaccharides, has a beneficial effect on microbiota composition. One small pilot study was performed to explore the effect of a combination treatment $(\mathrm{BCP}+B$. infantis $)$ in children ages $2-11$ with ASD and GI comorbidities. It has been indicated that the combination treatment is well-tolerated in this cohort and reduced GI symptoms and aberrant behaviors via inhibition of inflammatory factors (Sanctuary et al., 2019). One multi-center clinical study in China treated 37 children with ASD with 4 weeks of applied behavior analysis (ABA) training in combination with probiotics containing six strains of bacteria, while 28 other children with ASD were treated with ABA training alone (Niu et al., 2019). It has been confirmed that ABA training in combination with probiotics treatment brings more benefit to ASD children (Niu et al., 2019).

Metabolic modifications were also involved in the mechanism of probiotics for the treatment of ASD. One study from 58 autistic 
children and 39 healthy TD children found that GI symptoms were strongly correlated with the severity of autism. Probiotic oral supplementation decreased the levels of total SCFAs and lysozymes in ASD subjects (Adams et al., 2011). An openlabel trial found that in 22 autistic children (age range 4-10 years) oral supplementation with L. acidophilus strain Rosell-11 for 2 months decreased the level of d-arabinitol, a metabolite of Candida species, and improved the ability to concentrate and fulfil orders (Kałużna-Czaplińska and Błaszczyk, 2012).

\subsection{Antibiotics}

Studies have implicated that antibiotics alter the gut microbiota composition (Palleja et al., 2018). Antibiotic treatment disturbs existing microbial balance by inhibiting the growth of particular components of that ecosystem. Animal studies and clinical reports showed early-life exposure to antibiotics might be involved in the ASD pathogenesis (Holingue et al., 2020). On the other hand, pieces of evidence also suggest that antibiotics have potential benefits in ameliorating ASD symptoms (Xu et al., 2019).

\subsubsection{Treatment With Antibiotics in ASD Animals}

As mentioned above, $15 q$ dup mice display similar poor social communication and behavioral inflexibility to that observed in ASD patients. Neomycin treatment improved social communication in $15 q$ dup mice via increased beneficial OTUs such as Clostridium clusters XIVa and IV (Septyaningtrias et al., 2020). Interestingly, $k d m 5$-deficient flies displayed deficits in intestinal barrier and social behavior that correlate with compositional changes in the gut microbiota. Antibiotic treatment of conventionally reared $\mathrm{kdm} 5 \mathrm{~K} 6801 /$ 10424 flies and intestinal specific $k d m 5$ knockdown rescued social phenotypes and intestinal defects by modulating the gut microbiota (Chen et al., 2019). Moreover, antibiotic treatment of WT flies did not show social avoidance and social space differences in behavior (Chen et al., 2019).

\subsubsection{Treatment With Antibiotics in ASD Patients}

A prospective birth cohort with 116 ASD cases and 860 TD child controls found that the risk for ASD was attenuated in women who experienced MIA during pregnancy and received antibiotics, suggesting that antibiotic treatment during pregnancy in the context of MIA may protect against the increased risk for ASD in the offspring (Holingue et al., 2020). There is one case report on the administration of amoxicillin to five children diagnosed with an ASD and Lyme disease for 6 months, which deemed to improve speech, eye contact, sleep behaviors, and a reduction of repetitive behaviors (Kuhn et al., 2012).

In a pivotal study, Sandler et al. (2020) treated 11 regressivetype ASD children with oral vancomycin, a useful bactericidal antibiotic for treatment of Clostridium difficile-associated colitis, and noticed that communication and several behavioral defects were ameliorated markedly during the 8-week treatment period. However, these effects were transient, and behavior disorder appeared after vancomycin discontinuation to treat ASD children (Sandler et al., 2020). Additionally, vancomycin- resistant enterococcus is induced by overuse, a very serious health concern that should be considered, when vancomycin is applied to treat ASD children.

\subsection{FMT Therapy}

FMT is a promising approach for manipulating the gut microbiota by transferring donor fecal suspension to patients in order to correct dysbiosis of gut microbiota in the recipients. It has been proven successful in treating recurrent $C$. difficile infection (Xu et al., 2021), inflammatory bowel disease (Bauer et al., 2020; Tan et al., 2020), irritable bowel syndrome (Myneedu et al., 2019), and several kinds of non-GI diseases (Aroniadis and Brandt, 2013). FMT can effectively adjust the gut microbial profile and restore the proportion of anti-inflammatory bacteria (Ooijevaar et al., 2019). Therefore, establishing a healthy gut microbiota using FMT is a promising new treatment for ASD (Xu et al., 2021). Importantly, the FDA has granted approval to a FMT therapy applied in ASD children in 2019.

\subsubsection{Mice-Mice FMT}

Goo et al. (2020) found that FMT from normal mice to Fmr1 KO mice ameliorated autistic-like behaviors, especially in cognitive impairment and defects in social novelty preference. In addition, FMT reduced the increased levels of TNFa and Ibal in the Fmr1 KO mouse brains and normalized A. muciniphila level to WT level. Likewise, one recent study has reported that FMT from WT mice improved the autistic-like behaviors in EphB6 KO mice accompanied with the increased relative abundance of Deferribacteres at the phylum level and Mucispirillum at the genus level ( $\mathrm{Li}$ et al., 2020). In addition, decreased vitamin $\mathrm{B}_{6}$ in EphB6 KO mice is crucial for the gut microbiota-mediated autism-like behavior, which could be normalized by FMT from the WT mice (Li et al., 2020). Furthermore, abnormal social behaviors in GF mice are rescued after the mice are colonized with normal flora (Needham et al., 2018).

Studies have shown that social deficits and gut microbiota dysbiosis in MHFD offspring are inhibited by co-housing with offspring of mothers fed a regular diet (MRD) (Buffington et al., 2016). It has been reported that social behavior deficits were detected in GF mice due to lack of more bacterial species in the intestinal microbiota. FMT from MRD offspring at 4 weeks, but not at 8 weeks, rescued GF social behavior. This study indicates a critical neurodevelopmental window for microbial reconstruction and contributes to social behavior improvement (Needham et al., 2018).

\subsubsection{Human-Mice Inter-Species FMT}

On the other hand, the effect of FMT was evaluated in one recent study using cultured gut microbiota transplantation or conventional FMT from healthy individuals to the MIAinduced ASD mouse model (Chen et al., 2020). ASD mouse model displayed significant amelioration of anxiety-like and repetitive behaviors, as well as a correction of chemokine disorders (GRO- $\alpha$, MIP-1 $\alpha$, MCP-3, RANTES, and eotaxin) followed by transplantations with the original donor microbiota and the cultured microbiota (Chen et al., 2020). Moreover, both conventional FMT and the cultured 
microbiota transplantation have the ability to rescue several critical differential taxa (S24-7, Clostridiaceae, Prevotella, and Candidatus Arthromitus) in gut microbial composition of ASD mice, which is linked to serum levels of MIP-1a, MCP-3, RANTES, and eotaxin. It seemed that FMT from healthy human donors shifts the gut microbial profile in a mouse model of ASD closer to that of healthy mice (Chen et al., 2020).

\subsubsection{Human-Human FMT}

Obviously, FMT is likely to be a valuable treatment to correct dysbiotic gut microbiota in ASD patients through transferring opportunistic pathogens or infections. The clinical trial has been investigated to explore the influence of FMT on GI and behavior symptoms in 18 patients with ASD (7-16 years old) and comorbid GI symptoms (Kang et al., 2017). It has been revealed that 8-week FMT treatment improved most of the GI symptoms such as constipation, diarrhea, indigestion, and abdominal pain as well as ASD-related symptoms in 16 out of 18 ASD children (Kang et al., 2017). Different from vancomycin therapy, benefits produced by FMT were sustained at least 8 weeks after the treatment. Coincident with these clinical improvements, bacterial and phage deep sequencing analyses revealed that FMT increased the overall bacterial diversity and the potentially beneficial microbe abundance in the recipients, and these changes persisted for 8 weeks (Kang et al., 2017). These data indicate that FMT successfully shifts the gut microbiota of children with ASD toward that of healthy controls and their donors. This study sheds light on the potential of targeting gut microbiome for ASD treatment via restoring a healthy microbiota composition.

Moreover, a follow-up study at 2 years post-treatment was conducted and found that GI symptom improvements in most participants were maintained, and the autism symptoms had continued to improve since the end of treatment (Kang et al., 2019). Importantly, the gut microbial community diversity and the relative commensal bacterial abundances of two bacterial genera, Bifidobacteria and Prevotella, in ASD children were significantly increased after FMT treatment and remained pretty similar to those in TD children at 2 years after treatment (Kang et al., 2019). Besides, an open-label, randomized, wait-list-controlled trial performed by Zhao et al. (2019) showed that FMT treatment temporarily improved ASDrelated symptoms and GI symptoms 2 months following two FMTs in 24 ASD children compared to 24 control ASD children. Additionally, FMT therapy typically reduced the abundance of Bacteroides fragilis and persistently shaped the gut microbiota profile of ASD individuals to a healthy state.

Meanwhile, it has been reported that FMT treatment shifted plasma metabolite profiles in the ASD children to resemble more closely those of their TD peers (Kang et al., 2020). Likewise, in a recent study within a cohort of 18 ASD with GI symptoms and 20 TD children with no history of GI symptoms, FMT treatment induced global alterations in plasma profiles across diversified metabolic traits, such as nicotinate/nicotinamide and purine metabolism (Qureshi et al., 2020). In addition, FMT treatment caused driving the metabolic profile of the ASD group similar to the TD group. For 669 fecal metabolites detected, FMT treatment decreased $p$-Cresol sulfate levels in children with ASD similar to those in TD children (Qureshi et al., 2020). Due to striking heterogeneity in stool, the effect of FMT treatment on fecal metabolites will be re-valuated with a larger patient cohort and a placebo arm. Importantly, in a recent clinical trial involving 40 ASD (age 3-17 years) with GI symptoms and 16 TD children with no history of GI symptoms, FMT treatment mitigated autism symptoms and GI disorder, reconstructed gut microbiota, as well as recovered the serum levels of several neurotransmitters such as 5-HT, GABA, and DA in the ASD cohort (Li et al., 2021).

There are some challenges needed to be considered when FMT treatments were used to treat ASD. Dosage, duration of treatment, as well as use of antibiotics and bowel-cleansing regimes before treatment need to be determined with a larger population size and standardized clinical trial (Zhang et al., 2020). The first thing to be considered is that donors need to be screened before donation to minimize the risk of transferring opportunistic pathogens or infections to recipients.

\subsection{Dietary Interventions}

There is a growing body of scientific evidence that environmental factors such as diet affect GI microbiota composition. Therefore, dietary interventions may prove an easy approach to alter gut microbiota for neuropsychiatric patient treatment. Clinical trials have found that dietary patterns impact fecal microbiota composition and dynamic change in children with ASD (Berding and Donovan, 2018, 2020). Instead, one recent study indicated that ASD-related restricted behaviors might cause dietary restrictedness and in turn lead to decreased taxonomic diversity and looser stool consistency (Yap et al., 2021). The correlations between diet and fecal microbiota composition need to be further explored.

Vitamin D is essential for CNS development. Findings from previous studies have strengthened the link between vitamin D deficiency and the risk of autism. It has been reported that propionic acid occurs naturally in some foods and acts as a metabolic product of gut microbiota involved in the development of ASD. Alfawaz et al. (2014) further found that vitamin D displayed a greater protective than therapeutic effect on brain intoxication caused by propionic acid in rats. In line with this, a clinical study has indicated that vitamin D supplementation may alleviate symptoms of ASD when $25(\mathrm{OH}) \mathrm{D}$ levels in the serum were increased markedly (Javadfar et al., 2020). Polyunsaturated fatty acids (PUFAs) are important constituents of phospholipids. N-3 PUFA (docosahexaenoic acid) and n-6 PUFA (arachidonic acid) formed brain cellular membranes, are provided by dietary supply, and are critical for brain development evidenced by aggregation in embryonic and post-natal brains. Of note, evidence has shown that the state of $n-3$ and n-6 PUFAs also influences the gut microbiota, thereby improving autism-like behaviors. Wang et al. (2020a) also found the n-3/n-6 (1:5) diet improved fecal microbiota composition in VPA-exposed rats characterized by the increased microbial abundance and reduced Firmicutes. It is supposed that appropriate n-3/n-6 PUFA ratio intake is a promising intervention for treating ASD. Another study has also confirmed that orally supplemented 
omega-3 fatty acids and vitamin B12 combination were more efficacious in treating autism by improvements of oxidative stress and abnormal lipid metabolic as well as remodeling microbial communities (Alfawaz et al., 2018).

Furthermore, exclusion approaches, such as gluten- and casein-free diets (GFCF), had a favorable impact on ASDrelated symptoms. In one randomized clinical trial, intaking of GF diet improved GI symptom and ASD-related behavioral disorders (Ghalichi et al., 2016). A number of studies have addressed the beneficial effects of ketogenic diet composed of low carbohydrate, adequate protein, and high fat on improvement of autism-like behaviors via reshaping microbial composition (Newell et al., 2016; Rawat et al., 2021). There is still a lack of clinical data to support the correlation of observed behavioral improvements and GI symptoms produced by GFCF and ketogenic diet.

There are currently no randomized placebo-controlled clinical trials using dietary intervention, and this might be interpreted by the truth that individuals diagnosed with neuropsychiatric conditions intake different types of medications interfering with microbial communities. Although there are promising preliminary studies, further confirmative studies should be conducted to examine the extent by which past food habits of ASD patients and how the dietary interventions influence the pathogenesis and therapy of ASD.

\section{CONCLUSION AND CHALLENGES}

Despite discrepancies between studies, the close interaction of the gut microbiota with the physical condition of ASD patients indicates that abnormal microbiota composition may aggravate the behavioral symptoms and biological signs of ASD. Differences in microbiota composition remain uncertain due to differences in

\section{REFERENCES}

Aabed, K., Bhat, R. S., Al-Dbass, A., Moubayed, N., Algahtani, N., Merghani, N. M., et al. (2019). Bee Pollen and Propolis Improve Neuroinflammation and Dysbiosis Induced by Propionic Acid, a Short Chain Fatty Acid in a Rodent Model of Autism. Lipids Health Dis. 18, 200. doi:10.1186/s12944-019-1150-0

Adak, A., and Khan, M. R. (2019). An Insight into Gut Microbiota and its Functionalities. Cell. Mol. Life Sci. 76, 473-493. doi:10.1007/s00018-0182943-4

Adams, J. B., Johansen, L. J., Powell, L. D., Quig, D., and Rubin, R. A. (2011). Gastrointestinal flora and Gastrointestinal Status in Children with Autism Comparisons to Typical Children and Correlation with Autism Severity. BMC Gastroenterol. 11, 22. doi:10.1186/1471-230x-11-22

Adhya, D., Swarup, V., Nagy, R., Dutan, L., Shum, C., and Valencia-Alarcón, E. P. (2021). Atypical Neurogenesis in Induced Pluripotent Stem Cells from Autistic Individuals. Biol. Psychiatry 89, 486-496. doi:10.1016/j.biopsych. 2020.06.014

Al-Asmakh, M., and Hedin, L. (2015). Microbiota and the Control of BloodTissue Barriers. Tissue barriers 3, e1039691. doi:10.1080/21688370.2015. 1039691

Alfawaz, H. A., Bhat, R. S., Al-Ayadhi, L., and El-Ansary, A. K. (2014). Protective and Restorative Potency of Vitamin D on Persistent Biochemical Autistic Features Induced in Propionic Acid-Intoxicated Rat Pups. BMC Complement. Altern. Med. 14, 416. doi:10.1186/1472-6882-14-416 methodology, study population, and confounding factors. The manipulation of the gut microbiome seems to be a promising therapy to mitigate ASD-associated core symptoms and behavioral abnormalities in ASD subjects. Future studies should systematically investigate the microbial composition of children with ASD and stress the importance of interpreting a close association between typical bacterial species and ASD symptoms. Treatment using targeted bacterial strain is important, as the effects of probiotic bacteria can be highly strain specific. In addition, new therapeutic measures will be of key focus on providing an early intervention strategy to decrease the severity of the disease in ASD children. Longer follow-up of clinical course would help to further determine its efficacy and safety.

\section{AUTHOR CONTRIBUTIONS}

All authors contributed to the writing and editing of this review. $\mathrm{JL}$ and XF conceived the scope of the review, coordinated efforts among authors, and wrote the bulk of the microbiome and ASD sections. ZG, CL, TL and JG wrote the bulk of the treatment section. JL wrote the bulk of the pathogenesis mechanisms section. JL and YC aided in research and references to clinical studies. All authors contributed to the article and approved the submitted version.

\section{FUNDING}

This work was supported by the National Nature Science Foundation of China (No. 31871043) and the Natural Science Foundation Project of Chongqing (NO.cstc2020jcyjmsxmX0816).

Alfawaz, H., Al-Onazi, M., Bukhari, S. I., Binobead, M., Othman, N., Algahtani, N., et al. (2018). The Independent and Combined Effects of Omega-3 and Vitamin B12 in Ameliorating Propionic Acid Induced Biochemical Features in Juvenile Rats as Rodent Model of Autism. J. Mol. Neurosci. 66, 403-413. doi:10.1007/ s12031-018-1186-Z

Altimiras, F., Garcia, J. A., Palacios-García, I., Hurley, M. J., Deacon, R., González, B., et al. (2021). Altered Gut Microbiota in a Fragile X Syndrome Mouse Model. Front. Neurosci. 15, 653120. doi:10.3389/fnins.2021.653120

Angelucci, F., Cechova, K., Amlerova, J., and Hort, J. (2019). Antibiotics, Gut Microbiota, and Alzheimer's Disease. J. Neuroinflammation 16, 108. doi:10. 1186/s12974-019-1494-4

Arakawa, H. (2020). From Multisensory Assessment to Functional Interpretation of Social Behavioral Phenotype in Transgenic Mouse Models for Autism Spectrum Disorders. Front. Psychiatry 11, 592408. doi:10.3389/fpsyt.2020. 592408

Arnold, L. E., Luna, R. A., Williams, K., Chan, J., Parker, R. A., Wu, Q., et al. (2019). Probiotics for Gastrointestinal Symptoms and Quality of Life in Autism: A Placebo-Controlled Pilot Trial. J. Child. Adolesc. Psychopharmacol. 29, 659-669. doi:10.1089/cap.2018.0156

Aroniadis, O. C., and Brandt, L. J. (2013). Fecal Microbiota Transplantation: Past, Present and Future. Curr. Opin. Gastroenterol. 29, 79-84. doi:10.1097/mog. 0b013e32835a4b3e

Ashwood, P., Wills, S., and Van de Water, J. (2006). The Immune Response in Autism: a New Frontier for Autism Research. J. Leukoc. Biol. 80, 1-15. doi:10. 1189/jlb.1205707 
Baranova, J., Dragunas, G., Botellho, M. C. S., Ayub, A. L. P., Bueno-Alves, R., Alencar, R. R., et al. (2021). Autism Spectrum Disorder: Signaling Pathways and Prospective Therapeutic Targets. Cell Mol Neurobiol 41, 619-649. doi:10.1007/ s10571-020-00882-7

Bauer, C. M., Zhang, X., Long, M. D., and Sandler, R. S. (2020). Characteristics of Fecal Microbiota Transplantation Use in Inflammatory Bowel Disease Cohort. Crohns Colitis 360, otaa024. doi:10.1093/crocol/otaa024

Berding, K., and Donovan, S. M. (2020). Dietary Patterns Impact Temporal Dynamics of Fecal Microbiota Composition in Children with Autism Spectrum Disorder. Front. Nutr. 6, 193. doi:10.3389/fnut.2019.00193

Berding, K., and Donovan, S. M. (2018). Diet Can Impact Microbiota Composition in Children with Autism Spectrum Disorder. Front. Neurosci. 12, 515. doi:10. 3389/fnins.2018.00515

Bermudez-Martin, P., Becker, J. A. J., Caramello, N., Fernandez, S. P., CostaCampos, R., Canaguier, J., et al. (2021). The Microbial Metabolite P-Cresol Induces Autistic-like Behaviors in Mice by Remodeling the Gut Microbiota. Microbiome 9, 157. doi:10.1186/s40168-021-01103-Z

Bolte, E. R. (1998). Autism and Clostridium tetani. Med. Hypotheses 51, 133-144. doi:10.1016/s0306-9877(98)90107-4

Brunissen, L., Rapoport, E., Chawarska, K., and Adesman, A. (2021). Sex Differences in Gender-Diverse Expressions and Identities Among Youth with Autism Spectrum Disorder. Autism Res. 14, 143-155. doi:10.1002/aur. 2441

Buffington, S. A., Di Prisco, G. V., Auchtung, T. A., Ajami, N. J., Petrosino, J. F., and Costa-Mattioli, M. (2016). Microbial Reconstitution Reverses Maternal DietInduced Social and Synaptic Deficits in Offspring. Cell 165, 1762-1775. doi:10. 1016/j.cell.2016.06.001

Cai, Y., Tang, X., Chen, X., Li, X., Wang, Y., Bao, X., et al. (2018). Liver X Receptor $\beta$ Regulates the Development of the Dentate Gyrus and Autistic-like Behavior in the Mouse. Proc. Natl. Acad. Sci. U S A. 115, E2725-E2733. doi:10.1073/pnas. 1800184115

Cai, Y., Zhong, H., Li, X., Xiao, R., Wang, L., and Fan, X. (2019). The Liver X Receptor Agonist TO901317 Ameliorates Behavioral Deficits in Two Mouse Models of Autism. Front Cel Neurosci 13, 213. doi:10.3389/fncel.2019.00213

Cao, X., Liu, K., Liu, J., Liu, Y. W., Xu, L., Wang, H., et al. (2021). Dysbiotic Gut Microbiota and Dysregulation of Cytokine Profile in Children and Teens with Autism Spectrum Disorder. Front. Neurosci. 15, 635925. doi:10.3389/fnins. 2021.635925

Cast, T. P., Boesch, D. J., Smyth, K., Shaw, A. E., Ghebrial, M., and Chanda, S. (2021). An Autism-Associated Mutation Impairs Neuroligin-4 Glycosylation and Enhances Excitatory Synaptic Transmission in Human Neurons. J. Neurosci. 41, 392-407. doi:10.1523/jneurosci.0404-20.2020

Chen, K., Fu, Y., Wang, Y., Liao, L., Xu, H., Zhang, A., et al. (2020). Therapeutic Effects of the In Vitro Cultured Human Gut Microbiota as Transplants on Altering Gut Microbiota and Improving Symptoms Associated with Autism Spectrum Disorder. Microb. Ecol. 80, 475-486. doi:10.1007/s00248-02001494-w

Chen, K., Luan, X., Liu, Q., Wang, J., Chang, X., Snijders, A. M., et al. (2019). Drosophila Histone Demethylase KDM5 Regulates Social Behavior through Immune Control and Gut Microbiota Maintenance. Cell Host Microbe 25, 537-552. e8. doi:10.1016/j.chom.2019.02.003

Corbett, B. A., Ioannou, S., Key, A. P., Coke, C., Muscatello, R., Vandekar, S., et al. (2019). Treatment Effects in Social Cognition and Behavior Following a Theater-Based Intervention for Youth with Autism. Dev. Neuropsychol. 44, 481-494. doi:10.1080/87565641.2019.1676244

Coretti, L., Cristiano, C., Florio, E., Scala, G., Lama, A., Keller, S., et al. (2017). Sex-related Alterations of Gut Microbiota Composition in the BTBR Mouse Model of Autism Spectrum Disorder. Sci. Rep. 7, 45356. doi:10.1038/ srep45356

Coretti, L., Paparo, L., Riccio, M. P., Amato, F., Cuomo, M., Natale, A., et al. (2018). Gut Microbiota Features in Young Children with Autism Spectrum Disorders. Front. Microbiol. 9, 3146. doi:10.3389/fmicb.2018.03146

Cristiano, C., Pirozzi, C., Coretti, L., Cavaliere, G., Lama, A., Russo, R., et al. (2018). Palmitoylethanolamide Counteracts Autistic-like Behaviours in BTBR T+tf/J Mice: Contribution of central and Peripheral Mechanisms. Brain Behav. Immun. 74, 166-175. doi:10.1016/j.bbi.2018.09.003

Cristofori, F., Dargenio, V., Dargenio, C., Miniello, V., Barone, M., and Francavilla, R. (2021). Anti-Inflammatory and Immunomodulatory Effects of Probiotics in
Gut Inflammation: A Door to the Body. Front. Immunol. 12, 578386. doi:10. 3389/fimmu.2021.578386

Dan, Z., Mao, X., Liu, Q., Guo, M., Zhuang, Y., Liu, Z., et al. (2020). Altered Gut Microbial Profile Is Associated with Abnormal Metabolism Activity of Autism Spectrum Disorder. Gut Microbes 11, 1246-1267. doi:10.1080/19490976.2020. 1747329

Davies, C., Mishra, D., Eshraghi, R. S., Mittal, J., Sinha, R., Bulut, E., et al. (2021). Altering the Gut Microbiome to Potentially Modulate Behavioral Manifestations in Autism Spectrum Disorders: A Systematic Review. Neurosci. Biobehav Rev. 128, 549-557. doi:10.1016/j.neubiorev.2021.07.001

Dawson, L. F., Stabler, R. A., and Wren, B. W. (2008). Assessing the Role of P-Cresol Tolerance in Clostridium difficile. J. Med. Microbiol. 57, 745-749. doi:10.1099/jmm.0.47744-0

De Angelis, M., Francavilla, R., Piccolo, M., De Giacomo, A., and Gobbetti, M. (2015). Autism Spectrum Disorders and Intestinal Microbiota. Gut Microbes 6, 207-213. doi:10.1080/19490976.2015.1035855

de Theije, C. G., Wopereis, H., Ramadan, M., van Eijndthoven, T., Lambert, J., Knol, J., et al. (2014). Altered Gut Microbiota and Activity in a Murine Model of Autism Spectrum Disorders. Brain Behav. Immun. 37, 197-206. doi:10.1016/j. bbi.2013.12.005

Dhar, D., and Mohanty, A. (2020). Gut Microbiota and Covid-19- Possible Link and Implications. Virus. Res. 285, 198018. doi:10.1016/j.virusres.2020.198018

Dinan, T. G., and Cryan, J. F. (2017). The Microbiome-Gut-Brain Axis in Health and Disease. Gastroenterol. Clin. North. Am. 46, 77-89. doi:10.1016/j.gtc.2016. 09.007

Ding, X., Xu, Y., Zhang, X., Zhang, L., Duan, G., Song, C., et al. (2020). Gut Microbiota Changes in Patients with Autism Spectrum Disorders. J. Psychiatr. Res. 129, 149-159. doi:10.1016/j.jpsychires.2020.06.032

Donegan, J. J., and Lodge, D. J. (2020). Stem Cells for Improving the Treatment of Neurodevelopmental Disorders. Stem Cell Dev 29, 1118-1130. doi:10.1089/scd. 2019.0265

Dong, Z., Chen, W., Chen, C., Wang, H., Cui, W., Tan, Z., et al. (2020). CUL3 Deficiency Causes Social Deficits and Anxiety-like Behaviors by Impairing Excitation-Inhibition Balance through the Promotion of Cap-dependent Translation. Neuron 105, 475-490. e6. doi:10.1016/j.neuron.2019.10.035

Duerkop, B. A., Vaishnava, S., and Hooper, L. V. (2009). Immune Responses to the Microbiota at the Intestinal Mucosal Surface. Immunity 31, 368-376. doi:10. 1016/j.immuni.2009.08.009

El-Ansary, A., Bacha, A. B., Bjørklund, G., Al-Orf, N., Bhat, R. S., Moubayed, N., et al. (2018). Probiotic Treatment Reduces the Autistic-like Excitation/ inhibition Imbalance in Juvenile Hamsters Induced by Orally Administered Propionic Acid and Clindamycin. Metab. Brain Dis. 33, 1155-1164. doi:10. 1007/s11011-018-0212-8

Elsabbagh, M., Divan, G., Koh, Y. J., Kim, Y. S., Kauchali, S., Marcín, C., et al. (2012). Global Prevalence of Autism and Other Pervasive Developmental Disorders. Autism Res. 5, 160-179. doi:10.1002/aur.239

Engwa, G. A., Ayuk, E. L., Igbojekwe, B. U., and Unaegbu, M. (2016). Potential Antioxidant Activity of New Tetracyclic and Pentacyclic Nonlinear Phenothiazine Derivatives. Biochem. Res. Int. 2016, 9896575. doi:10.1155/ 2016/9896575

Ersöz Alan, B., and Gülerman, F. (2019). The Role of Gut Microbiota in Autism Spectrum Disorder. Turk Psikiyatri Derg 30, 210-219.

Finegold, S. M., Dowd, S. E., Gontcharova, V., Liu, C., Henley, K. E., Wolcott, R. D., et al. (2010). Pyrosequencing Study of Fecal Microflora of Autistic and Control Children. Anaerobe 16, 444-453. doi:10.1016/j.anaerobe.2010.06.008

Finegold, S. M., Molitoris, D., Song, Y., Liu, C., Vaisanen, M. L., Bolte, E., et al. (2002). Gastrointestinal Microflora Studies in Late-Onset Autism. Clin. Infect. Dis. 35, S6-S16. doi:10.1086/341914

Finegold, S. M. (2008). Therapy and Epidemiology of Autism-Clostridial Spores as Key Elements. Med. hypotheses 70, 508-511. doi:10.1016/j.mehy.2007.07.019

Frolli, A., Ricci, M. C., Tortorelli, F. A., Cavallaro, A., Valenzano, L., Rega, A., et al. (2020). Emotional Education in Early Onset Schizophrenia and Asperger's Syndrome. Behav. Sci. (Basel) 10, 131. doi:10.3390/bs10090131

Garcia-Gutierrez, E., Narbad, A., and Rodríguez, J. M. (2020). Autism Spectrum Disorder Associated with Gut Microbiota at Immune, Metabolomic, and Neuroactive Level. Front. Neurosci. 14, 578666. doi:10.3389/fnins.2020.578666

Ghalichi, F., Ghaemmaghami, J., Malek, A., and Ostadrahimi, A. (2016). Effect of Gluten Free Diet on Gastrointestinal and Behavioral Indices for Children with 
Autism Spectrum Disorders: a Randomized Clinical Trial. World J. Pediatr. 12, 436-442. doi:10.1007/s12519-016-0040-z

Giles, E. M., and Couper, J. (2020). Microbiome in Health and Disease. J. Paediatr. Child. Health 56, 1735-1738. doi:10.1111/jpc.14939

Golubeva, A. V., Joyce, S. A., Moloney, G., Burokas, A., Sherwin, E., Arboleya, S., et al. (2017). Microbiota-related Changes in Bile Acid \& Tryptophan Metabolism Are Associated with Gastrointestinal Dysfunction in a Mouse Model of Autism. EBioMedicine 24, 166-178. doi:10.1016/j.ebiom.2017. 09.020

Goo, N., Bae, H. J., Park, K., Kim, J., Jeong, Y., Cai, M., et al. (2020). The Effect of Fecal Microbiota Transplantation on Autistic-like Behaviors in Fmr1 KO Mice. Life Sci. 262, 118497. doi:10.1016/j.lfs.2020.118497

Gorrindo, P., Williams, K. C., Lee, E. B., Walker, L. S., McGrew, S. G., and Levitt, P. (2012). Gastrointestinal Dysfunction in Autism: Parental Report, Clinical Evaluation, and Associated Factors. Autism Res. 5, 101-108. doi:10.1002/ aur.237

Grimaldi, R., Gibson, G. R., Vulevic, J., Giallourou, N., Castro-Mejía, J. L., Hansen, L. H., et al. (2018). A Prebiotic Intervention Study in Children with Autism Spectrum Disorders (ASDs). Microbiome 6, 133. doi:10.1186/s40168-0180523-3

Grossi, E., Melli, S., Dunca, D., and Terruzzi, V. (2016). Unexpected Improvement in Core Autism Spectrum Disorder Symptoms after Long-Term Treatment with Probiotics. SAGE Open Med. Case Rep. 4, 2050313x16666231. doi:10.1177/ $2050313 \times 16666231$

Guo, Y., Huang, Z. P., Liu, C. Q., Qi, L., Sheng, Y., and Zou, D. J. (2018). Modulation of the Gut Microbiome: a Systematic Review of the Effect of Bariatric Surgery. Eur. J. Endocrinol. 178, 43-56. doi:10.1530/eje-17-0403

Ho, L. K. H., Tong, V. J. W., Syn, N., Nagarajan, N., Tham, E. H., Tay, S. K., et al. (2020). Gut Microbiota Changes in Children with Autism Spectrum Disorder: a Systematic Review. Gut Pathog. 12, 6. doi:10.1186/s13099-020-0346-1

Holingue, C., Brucato, M., Ladd-Acosta, C., Hong, X., Volk, H., Mueller, N. T., et al. (2020). Interaction between Maternal Immune Activation and Antibiotic Use during Pregnancy and Child Risk of Autism Spectrum Disorder. Autism Res. 13, 2230-2241. doi:10.1002/aur.2411

Hosie, S., Ellis, M., Swaminathan, M., Ramalhosa, F., Seger, G. O., Balasuriya, G. K., et al. (2019). Gastrointestinal Dysfunction in Patients and Mice Expressing the Autism-Associated R451C Mutation in Neuroligin-3. Autism Res. 12, 1043-1056. doi:10.1002/aur.2127

Hsiao, E. Y., McBride, S. W., Hsien, S., Sharon, G., Hyde, E. R., McCue, T., et al. (2013). Microbiota Modulate Behavioral and Physiological Abnormalities Associated with Neurodevelopmental Disorders. Cell 155, 1451-1463. doi:10. 1016/j.cell.2013.11.024

Jamain, S., Quach, H., Betancur, C., Råstam, M., Colineaux, C., Gillberg, I. C., et al. (2003). Mutations of the X-Linked Genes Encoding Neuroligins NLGN3 and NLGN4 Are Associated with Autism. Nat. Genet. 34, 27-29. doi:10.1038/ ng1136

Janmohammadi, P., Nourmohammadi, Z., Fazelian, S., Mirzababaei, A., Alizadeh, S., Zarei, M., et al. (2021). Does Infant Formula Containing Synbiotics Support Adequate Growth in Infants? A Meta-Analysis and Systematic Review of Randomized Controlled Trials. Crit. Rev. Food Sci. Nutr. 1-12, 1. doi:10. 1080/10408398.2021.1952548

Javadfar, Z., Abdollahzad, H., Moludi, J., Rezaeian, S., Amirian, H., Foroughi, A. A., et al. (2020). Effects of Vitamin D Supplementation on Core Symptoms, Serum Serotonin, and Interleukin-6 in Children with Autism Spectrum Disorders: A Randomized Clinical Trial. Nutrition 79-80 110986. doi:10.1016/j.nut.2020. 110986

Johnson, D., Letchumanan, V., Thurairajasingam, S., and Lee, L. H. (2020). A Revolutionizing Approach to Autism Spectrum Disorder Using the Microbiome. Nutrients 12, 1983. doi:10.3390/nu12071983

Kałużna-Czaplińska, J., and Błaszczyk, S. (2012). The Level of Arabinitol in Autistic Children after Probiotic Therapy. Nutrition 28, 124-126. doi:10.1016/j.nut. 2011.08.002

Kang, D. W., Adams, J. B., Coleman, D. M., Pollard, E. L., Maldonado, J., McDonough-Means, S., et al. (2019). Long-term Benefit of Microbiota Transfer Therapy on Autism Symptoms and Gut Microbiota. Sci. Rep. 9, 5821. doi:10.1038/s41598-019-42183-0

Kang, D. W., Adams, J. B., Gregory, A. C., Borody, T., Chittick, L., Fasano, A., et al. (2017). Microbiota Transfer Therapy Alters Gut Ecosystem and Improves
Gastrointestinal and Autism Symptoms: an Open-Label Study. Microbiome 5 10. doi:10.1186/s40168-016-0225-7

Kang, D. W., Adams, J. B., Vargason, T., Santiago, M., Hahn, J., and KrajmalnikBrown, R. (2020). Distinct Fecal and Plasma Metabolites in Children with Autism Spectrum Disorders and Their Modulation after Microbiota Transfer Therapy. mSphere 5, e00314-e00320. doi:10.1128/msphere.00314-20

Khan, S., Moore, R. J., Stanley, D., and Chousalkar, K. K. (2020). The Gut Microbiota of Laying Hens and its Manipulation with Prebiotics and Probiotics to Enhance Gut Health and Food Safety. Appl. Environ. Microbiol. 86, e00600-e00620. doi:10.1128/AEM.00600-20

Khatua, T. N., Borkar, R. M., Mohammed, S. A., Dinda, A. K., Srinivas, R., and Banerjee, S. K. (2017). Novel Sulfur Metabolites of Garlic Attenuate Cardiac Hypertrophy and Remodeling through Induction of $\mathrm{Na}^{+} / \mathrm{K}^{+}$-ATPase Expression. Front. Pharmacol. 8, 18. doi:10.3389/fphar.2017.00018

Kobliner, V., Mumper, E., and Baker, S. M. (2018). Reduction in Obsessive Compulsive Disorder and Self-Injurious Behavior with Saccharomyces Boulardii in a Child with Autism: A Case Report. Integr. Med. (Encinitas) $17,38-41$.

Kong, Q., Tian, P., Zhao, J., Zhang, H., Wang, G., and Chen, W. (2021a). The Autistic-like Behaviors Development during Weaning and Sexual Maturation in VPA-Induced Autistic-like Rats Is Accompanied by Gut Microbiota Dysbiosis. Peer J. 9, e11103. doi:10.7717/peerj.11103

Kong, Q., Wang, B., Tian, P., Li, X., Zhao, J., Zhang, H., et al. (2021b). Daily Intake of Lactobacillus Alleviates Autistic-like Behaviors by Ameliorating the 5hydroxytryptamine Metabolic Disorder in VPA-Treated Rats during Weaning and Sexual Maturation. Food Funct. 12, 2591-2604. doi:10.1039/ d0fo02375b

Kong, X. J., Liu, J., Liu, K., Koh, M., Sherman, H., Liu, S., et al. (2021c). Probiotic and Oxytocin Combination Therapy in Patients with Autism Spectrum Disorder: A Randomized, Double-Blinded, Placebo-Controlled Pilot Trial. Nutrients 13, 1552. doi:10.3390/nu13051552

Kuhn, M., Grave, S., Bransfield, R., and Harris, S. (2012). Long Term Antibiotic Therapy May Be an Effective Treatment for Children Co-morbid with Lyme Disease and Autism Spectrum Disorder. Med. hypotheses 78, 606-615. doi:10. 1016/j.mehy.2012.01.037

Lasheras, I., Seral, P., Latorre, E., Barroso, E., Gracia-García, P., and Santabárbara, J. (2020). Microbiota and Gut-Brain axis Dysfunction in Autism Spectrum Disorder: Evidence for Functional Gastrointestinal Disorders. Asian J. Psychiatr. 47, 101874. doi:10.1016/j.ajp.2019.101874

Lefter, R., Ciobica, A., Timofte, D., Stanciu, C., and Trifan, A. (2019). A Descriptive Review on the Prevalence of Gastrointestinal Disturbances and Their Multiple Associations in Autism Spectrum Disorder. Medicina (Kaunas, Lithuania) 56, 11. doi:10.3390/medicina56010011

Li, N., Chen, H., Cheng, Y., Xu, F., Ruan, G., Ying, S., et al. (2021). Fecal Microbiota Transplantation Relieves Gastrointestinal and Autism Symptoms by Improving the Gut Microbiota in an Open-Label Study. Front Cel Infect Microbiol 11, 759435. doi: $10.3389 /$ fcimb. 2021.759435

Li, Q., Han, Y., Dy, A., and Hagerman, R. J. (2017). The Gut Microbiota and Autism Spectrum Disorders. Front. Cel Neurosci 11, 120. doi:10.3389/fncel. 2017.00120

Li, Y., Luo, Z. Y., Hu, Y. Y., Bi, Y. W., Yang, J. M., Zou, W. J., et al. (2020). The Gut Microbiota Regulates Autism-like Behavior by Mediating Vitamin B(6) Homeostasis in EphB6-Deficient Mice. Microbiome 8, 120. doi:10.1186/ s40168-020-00884-z

Liu, F., Horton-Sparks, K., Hull, V., Li, R. W., and Martínez-Cerdeño, V. (2018). The Valproic Acid Rat Model of Autism Presents with Gut Bacterial Dysbiosis Similar to that in Human Autism. Mol. autism 9, 61. doi:10.1186/s13229-0180251-3

Liu, Y. W., Liong, M. T., Chung, Y. E., Huang, H. Y., Peng, W. S., Cheng, Y. F., et al. (2019). Effects of Lactobacillus Plantarum PS128 on Children with Autism Spectrum Disorder in Taiwan: A Randomized, Double-Blind, PlaceboControlled Trial. Nutrients 11, 820. doi:10.3390/nu11040820

Liu, Z., Mao, X., Dan, Z., Pei, Y., Xu, R., Guo, M., et al. (2021). Gene Variations in Autism Spectrum Disorder Are Associated with Alteration of Gut Microbiota, Metabolites and Cytokines. Gut Microbes 13, 1-16. doi:10.1080/19490976.2020. 1854967

Lombardi, V. C., De Meirleir, K. L., Subramanian, K., Nourani, S. M., Dagda, R. K., Delaney, S. L., et al. (2018). Nutritional Modulation of the Intestinal Microbiota; 
Future Opportunities for the Prevention and Treatment of Neuroimmune and Neuroinflammatory Disease. J. Nutr. Biochem. 61, 1-16. doi:10.1016/j.jnutbio. 2018.04.004

Luna, R. A., Oezguen, N., Balderas, M., Venkatachalam, A., Runge, J. K., Versalovic, J., et al. (2017). Distinct Microbiome-Neuroimmune Signatures Correlate with Functional Abdominal Pain in Children with Autism Spectrum Disorder. Cell Mol Gastroenterol Hepatol 3, 218-230. doi:10.1016/j.jcmgh.2016.11.008

Ma, Y., Liu, T., Fu, J., Fu, S., Hu, C., Sun, B., et al. (2019). Lactobacillus Acidophilus Exerts Neuroprotective Effects in Mice with Traumatic Brain Injury. J. Nutr. 149, 1543-1552. doi:10.1093/jn/nxz105

Macfabe, D. F. (2012). Short-chain Fatty Acid Fermentation Products of the Gut Microbiome: Implications in Autism Spectrum Disorders. Microb. Ecol. Health Dis. 23, 1. doi:10.3402/mehd.v23i0.19260

Maigoro, A. Y., and Lee, S. (2021). Gut Microbiome-Based Analysis of Lipid A Biosynthesis in Individuals with Autism Spectrum Disorder: An In Silico Evaluation. Nutrients 13, 688. doi:10.3390/nu13020688

Martínez-González, A. E., and Andreo-Martínez, P. (2020). Prebiotics, Probiotics and Fecal Microbiota Transplantation in Autism: A Systematic Review. Revista de psiquiatria y salud Ment. 13, 150-164. doi:10.1016/j.rpsm.2020.06.002

Mensi, M. M., Rogantini, C., Marchesi, M., Borgatti, R., and Chiappedi, M. (2021). Lactobacillus Plantarum PS128 and Other Probiotics in Children and Adolescents with Autism Spectrum Disorder: A Real-World Experience. Nutrients 13, 688. doi:10.3390/nu13062036

Moszak, M., Szulińska, M., and Bogdański, P. (2020). You Are what You Eat-The Relationship between Diet, Microbiota, and Metabolic Disorders-A Review. Nutrients 12, 1096. doi:10.3390/nu12041096

Myneedu, K., Deoker, A., Schmulson, M. J., and Bashashati, M. (2019). Fecal Microbiota Transplantation in Irritable Bowel Syndrome: A Systematic Review and Meta-Analysis. United Eur. Gastroenterol J 7, 1033-1041. doi:10.1177/ 2050640619866990

Needham, B. D., Tang, W., and Wu, W. L. (2018). Searching for the Gut Microbial Contributing Factors to Social Behavior in Rodent Models of Autism Spectrum Disorder. Dev. Neurobiol. 78, 474-499. doi:10.1002/dneu.22581

Neuhaus, E., Bernier, R. A., Tham, S. W., and Webb, S. J. (2018). Gastrointestinal and Psychiatric Symptoms Among Children and Adolescents with Autism Spectrum Disorder. Front. Psychiatry 9, 515. doi:10.3389/fpsyt.2018.00515

Newell, C., Bomhof, M. R., Reimer, R. A., Hittel, D. S., Rho, J. M., and Shearer, J. (2016). Ketogenic Diet Modifies the Gut Microbiota in a Murine Model of Autism Spectrum Disorder. Mol. Autism 7, 37. doi:10.1186/s13229-016-0099-3

Niu, M., Li, Q., Zhang, J., Wen, F., Dang, W., Duan, G., et al. (2019). Characterization of Intestinal Microbiota and Probiotics Treatment in Children with Autism Spectrum Disorders in China. Front. Neurol. 10, 1084. doi:10.3389/fneur.2019.01084

O’Donnell, M. P., Fox, B. W., Chao, P. H., Schroeder, F. C., and Sengupta, P. (2020). A Neurotransmitter Produced by Gut Bacteria Modulates Host Sensory Behaviour. Nature 583, 415-420. doi:10.1038/s41586-020-2395-5

Ogunrinola, G. A., Oyewale, J. O., Oshamika, O. O., and Olasehinde, G. I. (2020). The Human Microbiome and its Impacts on Health. Int. J. Microbiol. 2020, 8045646. doi:10.1155/2020/8045646

Oh, D., and Cheon, K. A. (2020). Alteration of Gut Microbiota in Autism Spectrum Disorder: An Overview. Soa Chongsonyon Chongsin Uihak 31, 131-145. doi:10. 5765/jkacap.190039

Ooijevaar, R. E., Terveer, E. M., Verspaget, H. W., Kuijper, E. J., and Keller, J. J. (2019). Clinical Application and Potential of Fecal Microbiota Transplantation. Annu. Rev. Med. 70, 335-351. doi:10.1146/annurev-med-111717-122956

Oshima, F., William, M., Takahashi, N., Tsuchiyagaito, A., Kuwabara, H., Shiina, A., et al. (2020). Cognitive-behavioral Family Therapy as Psychoeducation for Adolescents with High-Functioning Autism Spectrum Disorders: Aware and Care for My Autistic Traits (ACAT) Program Study Protocol for a Pragmatic Multisite Randomized Controlled Trial. Trials 21, 814. doi:10.1186/s13063020-04750-z

Palleja, A., Mikkelsen, K. H., Forslund, S. K., Kashani, A., Allin, K. H., Nielsen, T., et al. (2018). Recovery of Gut Microbiota of Healthy Adults Following Antibiotic Exposure. Nat. Microbiol. 3, 1255-1265. doi:10.1038/s41564-0180257-9

Parada Venegas, D., De la Fuente, M. K., Landskron, G., González, M. J., Quera, R., Dijkstra, G., et al. (2019). Short Chain Fatty Acids (SCFAs)-Mediated Gut
Epithelial and Immune Regulation and its Relevance for Inflammatory Bowel Diseases. Front. Immunol. 10, 277. doi:10.3389/fimmu.2019.00277

Parracho, H. M., Bingham, M. O., Gibson, G. R., and McCartney, A. L. (2005). Differences between the Gut Microflora of Children with Autistic Spectrum Disorders and that of Healthy Children. J. Med. Microbiol. 54, 987-991. doi:10. 1099/jmm.0.46101-0

Pellegrini, C., Antonioli, L., Calderone, V., Colucci, R., Fornai, M., and Blandizzi, C. (2020). Microbiota-gut-brain axis in Health and Disease: Is NLRP3 Inflammasome at the Crossroads of Microbiota-Gut-Brain Communications? Prog. Neurobiol. 191, 101806. doi:10.1016/j.pneurobio. 2020.101806

Poornimai Abirami, G. P., Radhakrishnan, R. K., Johnson, E., Roshan, S. A., Yesudhas, A., Parveen, S., et al. (2020). The Regulation of Reactive Neuroblastosis, Neuroplasticity, and Nutraceuticals for Effective Management of Autism Spectrum Disorder. Adv. Neurobiol. 24, 207-222. doi:10.1007/978-3-030-30402-7_8

Qi, Z., Lyu, M., Yang, L., Yuan, H., Cao, Y., Zhai, L., et al. (2021). A Novel and Reliable Rat Model of Autism. Front. Psychiatry 12, 549810. doi:10.3389/fpsyt. 2021.549810

Qureshi, F., Adams, J., Hanagan, K., Kang, D. W., Krajmalnik-Brown, R., and Hahn, J. (2020). Multivariate Analysis of Fecal Metabolites from Children with Autism Spectrum Disorder and Gastrointestinal Symptoms before and after Microbiota Transfer Therapy. J. Pers Med. 10, 152. doi:10.3390/ jpm10040152

Rajkumar, H., Mahmood, N., Kumar, M., Varikuti, S. R., Challa, H. R., and Myakala, S. P. (2014). Effect of Probiotic (VSL\#3) and omega-3 on Lipid Profile, Insulin Sensitivity, Inflammatory Markers, and Gut Colonization in Overweight Adults: a Randomized, Controlled Trial. Mediators Inflamm. 2014, 348959. doi:10.1155/2014/348959

Rautava, S., Luoto, R., Salminen, S., and Isolauri, E. (2012). Microbial Contact during Pregnancy, Intestinal Colonization and Human Disease. Nat. Rev. Gastroenterol. Hepatol. 9, 565-576. doi:10.1038/nrgastro.2012.144

Rawat, K., Singh, N., Kumari, P., and Saha, L. (2021). A Review on Preventive Role of Ketogenic Diet (KD) in CNS Disorders from the Gut Microbiota Perspective. Rev. Neurosci. 32, 143-157. doi:10.1515/revneuro-2020-0078

Reigstad, C. S., Salmonson, C. E., Rainey, J. F., 3rd., Szurszewski, J. H., Linden, D. R., Sonnenburg, J. L., et al. (2015). Gut Microbes Promote Colonic Serotonin Production through an Effect of Short-Chain Fatty Acids on Enterochromaffin Cells. FASEB J. 29, 1395-1403. doi:10.1096/fj.14-259598

Riccio, P., and Rossano, R. (2019). Undigested Food and Gut Microbiota May Cooperate in the Pathogenesis of Neuroinflammatory Diseases: A Matter of Barriers and a Proposal on the Origin of Organ Specificity. Nutrients 11, 2714. doi:10.3390/nu11112714

Romagnoli, G., Leone, A., Romagnoli, G., Sansoni, J., Tofani, M., De Santis, R., et al. (2019). Occupational Therapy's Efficacy in Children with Asperger's Syndrome: a Systematic Review of Randomized Controlled Trials. Clin. Ter 170, e382-e387. doi:10.7417/CT.2019.2164

Sacai, H., Sakoori, K., Konno, K., Nagahama, K., Suzuki, H., Watanabe, T., et al. (2020). Autism Spectrum Disorder-like Behavior Caused by Reduced Excitatory Synaptic Transmission in Pyramidal Neurons of Mouse Prefrontal Cortex. Nat. Commun. 11, 5140. doi:10.1038/s41467-020-18861-3

Sakkas, H., Bozidis, P., Touzios, C., Kolios, D., Athanasiou, G., Athanasopoulou, E., et al. (2020). Nutritional Status and the Influence of the Vegan Diet on the Gut Microbiota and Human Health. Medicina (Kaunas, Lithuania) 56, 88. doi:10. 3390/medicina56020088

Sanctuary, M. R., Kain, J. N., Chen, S. Y., Kalanetra, K., Lemay, D. G., Rose, D. R., et al. (2019). Pilot Study of Probiotic/colostrum Supplementation on Gut Function in Children with Autism and Gastrointestinal Symptoms. PloS one 14, e0210064. doi:10.1371/journal.pone.0210064

Sandler, R. H., Finegold, S. M., Bolte, E. R., Buchanan, C. P., Maxwell, A. P., Väisänen, M. L., et al. (2020). Short-term Benefit from Oral Vancomycin Treatment of Regressive-Onset Autism. J. Child. Neurol. 15, 429-435. doi:10. 1177/088307380001500701

Santocchi, E., Guiducci, L., Fulceri, F., Billeci, L., Buzzigoli, E., Apicella, F., et al. (2016). Gut to Brain Interaction in Autism Spectrum Disorders: a Randomized Controlled Trial on the Role of Probiotics on Clinical, Biochemical and Neurophysiological Parameters. BMC psychiatry 16, 183. doi:10.1186/ s12888-016-0887-5 
Santocchi, E., Guiducci, L., Prosperi, M., Calderoni, S., Gaggini, M., Apicella, F., et al. (2020). Effects of Probiotic Supplementation on Gastrointestinal, Sensory and Core Symptoms in Autism Spectrum Disorders: A Randomized Controlled Trial. Front. Psychiatry 11, 550593. doi:10.3389/fpsyt.2020.550593

Seitz, J., Dahmen, B., Keller, L., and Herpertz-Dahlmann, B. (2020). Gut Feelings: How Microbiota Might Impact the Development and Course of Anorexia Nervosa. Nutrients 12, 3295. doi:10.3390/nu12113295

Selimbeyoglu, A., Kim, C. K., Inoue, M., Lee, S. Y., Hong, A. S. O., Kauvar, I., et al. (2017). Modulation of Prefrontal Cortex Excitation/inhibition Balance Rescues Social Behavior in CNTNAP2-Deficient Mice. Sci. Transl Med. 9, eaah6733. doi:10.1126/scitranslmed.aah6733

Septyaningtrias, D. E., Lin, C. W., Ouchida, R., Nakai, N., Suda, W., Hattori, M., et al. (2020). Altered Microbiota Composition Reflects Enhanced Communication in 15q11-13 CNV Mice. Neurosci. Res. 161, 59-67. doi:10. 1016/j.neures.2019.12.010

Sgritta, M., Dooling, S. W., Buffington, S. A., Momin, E. N., Francis, M. B., Britton, R. A., et al. (2019). Mechanisms Underlying Microbial-Mediated Changes in Social Behavior in Mouse Models of Autism Spectrum Disorder. Neuron 101, 246e246-259. doi:10.1016/j.neuron.2018.11.018

Shaaban, S. Y., El Gendy, Y. G., Mehanna, N. S., El-Senousy, W. M., El-Feki, H. S. A., Saad, K., et al. (2018). The Role of Probiotics in Children with Autism Spectrum Disorder: A Prospective, Open-Label Study. Nutr. Neurosci. 21, 676-681. doi:10.1080/1028415x.2017.1347746

Shanahan, F., Ghosh, T. S., and O'Toole, P. W. (2021). The Healthy MicrobiomeWhat Is the Definition of a Healthy Gut Microbiome? Gastroenterology 160, 483-494. doi:10.1053/j.gastro.2020.09.057

Sivamaruthi, B. S., Suganthy, N., Kesika, P., and Chaiyasut, C. (2020). The Role of Microbiome, Dietary Supplements, and Probiotics in Autism Spectrum Disorder. Int. J. Environ. Res. Public Health 17, 2647. doi:10.3390/ ijerph 17082647

Song, Y., Liu, C., and Finegold, S. M. (2004). Real-time PCR Quantitation of Clostridia in Feces of Autistic Children. Appl. Environ. Microbiol. 70, 6459-6465. doi:10.1128/aem.70.11.6459-6465.2004

Stilling, R. M., Moloney, G. M., Ryan, F. J., Hoban, A. E., Bastiaanssen, T. F., Shanahan, F., et al. (2018). Social Interaction-Induced Activation of RNA Splicing in the Amygdala of Microbiome-Deficient Mice. Elife 7, e33070. doi:10. 7554/eLife.33070

Stilling, R. M., Ryan, F. J., Hoban, A. E., Shanahan, F., Clarke, G., Claesson, M. J., et al. (2015). Microbes \& Neurodevelopment--Absence of Microbiota during Early Life Increases Activity-Related Transcriptional Pathways in the Amygdala. Brain Behav. Immun. 50, 209-220. doi:10.1016/j.bbi.2015.07.009

Sun, H., You, Z., Jia, L., and Wang, F. (2019). Autism Spectrum Disorder Is Associated with Gut Microbiota Disorder in Children. BMC Pediatr. 19, 516. doi:10.1186/s12887-019-1896-6

Supekar, K., Ryali, S., Mistry, P., and Menon, V. J. N. C. (2021). Aberrant Dynamics of Cognitive Control and Motor Circuits Predict Distinct Restricted and Repetitive Behaviors in Children with Autism. Nat. Commun. 12, 3537. doi:10.1038/s41467-021-23822-5

Tabouy, L., Getselter, D., Ziv, O., Karpuj, M., Tabouy, T., Lukic, I., et al. (2018). Dysbiosis of Microbiome and Probiotic Treatment in a Genetic Model of Autism Spectrum Disorders. Brain Behav. Immun. 73, 310-319. doi:10.1016/j. bbi.2018.05.015

Tan, P., Li, X., Shen, J., and Feng, Q. (2020). Fecal Microbiota Transplantation for the Treatment of Inflammatory Bowel Disease: An Update. Front. Pharmacol. 11, 574533. doi:10.3389/fphar.2020.574533

Tomova, A., Husarova, V., Lakatosova, S., Bakos, J., Vlkova, B., Babinska, K., et al. (2015). Gastrointestinal Microbiota in Children with Autism in Slovakia. Physiol. Behav. 138, 179-187. doi:10.1016/j.physbeh.2014.10.033

Tran, S. M., and Mohajeri, M. H. (2021). The Role of Gut Bacterial Metabolites in Brain Development. Aging and DiseaseNutrients 13, 732. doi:10.3390/ nu13030732

Travagli, R. A., Browning, K. N., and Camilleri, M. (2020). Parkinson Disease and the Gut: New Insights into Pathogenesis and Clinical Relevance. Nat. Rev. Gastroenterol. Hepatol. 17, 673-685. doi:10.1038/s41575-020-0339-z

Valentino, F., Bruno, L., Doddato, G., Giliberti, A., Tita, R., Resciniti, S., et al. (2021). Exome Sequencing in 200 Intellectual Disability/Autistic Patients: New Candidates and Atypical Presentations. Brain Sci. 11, 936. doi:10.3390/ brainsci1 1070936
Vargason, T., McGuinness, D. L., and Hahn, J. (2019). Gastrointestinal Symptoms and Oral Antibiotic Use in Children with Autism Spectrum Disorder: Retrospective Analysis of a Privately Insured U.S. Population. J. Autism Dev. Disord. 49, 647-659. doi:10.1007/s10803-018-3743-2

Vuong, H. E., and Hsiao, E. Y. (2017). Emerging Roles for the Gut Microbiome in Autism Spectrum Disorder. Biol. Psychiatry 81, 411-423. doi:10.1016/j. biopsych.2016.08.024

Wan, Y., Zuo, T., Xu, Z., Zhang, F., Zhan, H., Chan, D., et al. (2021). Underdevelopment of the Gut Microbiota and Bacteria Species as Noninvasive Markers of Prediction in Children with Autism Spectrum Disorder. Gut 2020, 324015. doi:10.1136/gutjnl-2020-324015

Wang, J. P., Xu, Y. C., Hou, J. Q., Li, J. Y., Xing, J., Yang, B. X., et al. (2020a). Effects of Dietary Fat Profile on Gut Microbiota in Valproate Animal Model of Autism. Front. Med. 7, 151. doi:10.3389/fmed.2020.00151

Wang, L. W., Tancredi, D. J., and Thomas, D. W. (2011). The Prevalence of Gastrointestinal Problems in Children across the United States with Autism Spectrum Disorders from Families with Multiple Affected Members. J. Dev. Behav. Pediatr. 32, 351-360. doi:10.1097/dbp.0b013e31821bd06a

Wang, X., Yang, J., Zhang, H., Yu, J., and Yao, Z. (2019). Oral Probiotic Administration during Pregnancy Prevents Autism-Related Behaviors in Offspring Induced by Maternal Immune Activation via Anti-inflammation in Mice. Autism Res. 12, 576-588. doi:10.1002/aur.2079

Wang, Y., Li, N., Yang, J. J., Zhao, D. M., Chen, B., Zhang, G. Q., et al. (2020b). Probiotics and Fructo-Oligosaccharide Intervention Modulate the MicrobiotaGut Brain axis to Improve Autism Spectrum Reducing Also the HyperSerotonergic State and the Dopamine Metabolism Disorder. Pharmacol. Res. 157, 104784. doi:10.1016/j.phrs.2020.104784

Williams, B. L., Hornig, M., Buie, T., Bauman, M. L., Cho Paik, M., Wick, I., et al. (2011). Impaired Carbohydrate Digestion and Transport and Mucosal Dysbiosis in the Intestines of Children with Autism and Gastrointestinal Disturbances. PloS one 6, e24585. doi:10.1371/journal.pone.0024585

Xiao, L., Yan, J., Yang, T., Zhu, J., Li, T., Wei, H., et al. (2021). Fecal Microbiome Transplantation from Children with Autism Spectrum Disorder Modulates Tryptophan and Serotonergic Synapse Metabolism and Induces Altered Behaviors in Germ-free Mice. mSystems 6, e01343. doi:10.1128/mSystems. 01343-20

Xu, H. M., Huang, H. L., Zhou, Y. L., Zhao, H. L., Xu, J., Shou, D. W., et al. (2021). Fecal Microbiota Transplantation: A New Therapeutic Attempt from the Gut to the Brain. Gastroenterol. Res. Pract. 2021, 6699268. doi:10.1155/2021/6699268

Xu, M., Xu, X., Li, J., and Li, F. (2019). Association between Gut Microbiota and Autism Spectrum Disorder: A Systematic Review and Meta-Analysis. Front. Psychiatry 10, 473. doi:10.3389/fpsyt.2019.00473

Yang, J., Fu, X., Liao, X., and Li, Y. (2020). Effects of Gut Microbial-Based Treatments on Gut Microbiota, Behavioral Symptoms, and Gastrointestinal Symptoms in Children with Autism Spectrum Disorder: A Systematic Review. Psychiatry Res. 293, 113471. doi:10.1016/j.psychres.2020.113471

Yano, J. M., Yu, K., Donaldson, G. P., Shastri, G. G., Ann, P., Ma, L., et al. (2015). Indigenous Bacteria from the Gut Microbiota Regulate Host Serotonin Biosynthesis. Cell 161, 264-276. doi:10.1016/j.cell.2015.02.047

Yap, C. X., Henders, A. K., Alvares, G. A., Wood, D. L. A., Krause, L., Tyson, G. W., et al. (2021). Autism-related Dietary Preferences Mediate Autism-Gut Microbiome Associations. Cell 184, 5916-5931. doi:10.1016/j.cell.2021. 10.015

Yoo, J. Y., Groer, M., Dutra, S. V. O., Sarkar, A., and McSkimming, D. I. (2020). Gut Microbiota and Immune System Interactions. Microorganisms 8. doi:10.3390/ microorganisms 8101587

Żebrowska, P., Łaczmańska, I., and Łaczmański, Ł. (2021). Future Directions in Reducing Gastrointestinal Disorders in Children with ASD Using Fecal Microbiota Transplantation. Front. Cel Infect Microbiol 11, 630052. doi:10. 3389/fcimb.2021.630052

Zhang, M., Ma, W., Zhang, J., He, Y., and Wang, J. (2018). Analysis of Gut Microbiota Profiles and Microbe-Disease Associations in Children with Autism Spectrum Disorders in China. Sci. Rep. 8, 13981. doi:10.1038/s41598-018$32219-2$

Zhang, R., Cai, Y., Xiao, R., Zhong, H., Li, X., Guo, L., et al. (2019). Human Amniotic Epithelial Cell Transplantation Promotes Neurogenesis and Ameliorates Social Deficits in BTBR Mice. Stem Cel Res Ther 10, 153 doi:10.1186/s13287-019-1267-0 
Zhang, T., Lu, G., Zhao, Z., Liu, Y., Shen, Q., Li, P., et al. (2020). Washed Microbiota Transplantation vs. Manual Fecal Microbiota Transplantation: Clinical Findings, Animal Studies and In Vitro Screening. Protein Cell 11, 251-266. doi:10.1007/s13238-019-00684-8

Zhang, Y., Bitner, D., Pontes Filho, A. A., Li, F., Liu, S., Wang, H., et al. (2014). Expression and Function of NIK- and IKK2-Binding Protein (NIBP) in Mouse Enteric Nervous System. Neurogastroenterol Motil. 26, 77-97. doi:10.1111/ nmo.12234

Zhang, Y., Qu, Y., Yang, J., Liu, J., Li, S., and He, X. (2021). A Pilot Study to Investigate the Alteration of Gut Microbial Profile in Dip2a Knockout Mice. Int. Microbiol. 1, 1. doi:10.1007/s10123-021-00211-y

Zhao, H., Gao, X., Xi, L., Shi, Y., Peng, L., Wang, C., et al. (2019). Mo1667 Fecal Microbiota Transplantation for Children with Autism Spectrum Disorder. Gastrointest. Endos copy 89, AB512-AB513. doi:10.1016/j.gie. 2019.03.857

Zhong, H., Xiao, R., Ruan, R., Liu, H., Li, X., Cai, Y., et al. (2020). Neonatal Curcumin Treatment Restores Hippocampal Neurogenesis and Improves Autism-Related Behaviors in a Mouse Model of Autism. Psychopharmacology 237, 3539-3552. doi:10.1007/s00213020-05634-5

Zhu, C. S., Grandhi, R., Patterson, T. T., and Nicholson, S. E. (2018). A Review of Traumatic Brain Injury and the Gut Microbiome: Insights into Novel Mechanisms of Secondary Brain Injury and Promising
Targets for Neuroprotection. Brain Sci. 8, 113. doi:10.3390/ brainsci8060113

Zou, R., Xu, F., Wang, Y., Duan, M., Guo, M., Zhang, Q., et al. (2020). Changes in the Gut Microbiota of Children with Autism Spectrum Disorder. Autism Res. 13, 1614-1625. doi:10.1002/aur.2358

Conflict of Interest: The authors declare that the research was conducted in the absence of any commercial or financial relationships that could be construed as a potential conflict of interest.

Publisher's Note: All claims expressed in this article are solely those of the authors and do not necessarily represent those of their affiliated organizations, or those of the publisher, the editors and the reviewers. Any product that may be evaluated in this article, or claim that may be made by its manufacturer, is not guaranteed or endorsed by the publisher.

Copyright (C) $2022 \mathrm{Liu}, \mathrm{Gao}, \mathrm{Liu}, \mathrm{Liu}, \mathrm{Gao}, \mathrm{Cai}$ and Fan. This is an open-access article distributed under the terms of the Creative Commons Attribution License (CC BY). The use, distribution or reproduction in other forums is permitted, provided the original author(s) and the copyright owner(s) are credited and that the original publication in this journal is cited, in accordance with accepted academic practice. No use, distribution or reproduction is permitted which does not comply with these terms. 